\title{
Differential optical absorption spectroscopy (DOAS) and air mass factor concept for a multiply scattering vertically inhomogeneous medium: theoretical consideration
}

\author{
V. V. Rozanov and A. V. Rozanov \\ Institute of Environmental Physics (IUP), University of Bremen, Bremen, Germany \\ Received: 18 January 2010 - Published in Atmos. Meas. Tech. Discuss.: 17 February 2010 \\ Revised: 14 May 2010 - Accepted: 9 June 2010 - Published: 29 June 2010
}

\begin{abstract}
The Differential Optical Absorption Spectroscopy (DOAS) technique is widely used to retrieve amounts of atmospheric species from measurements of the direct solar light transmitted through the Earth's atmosphere as well as of the solar light scattered in the atmosphere or reflected from the Earth's surface. For the transmitted direct solar light the theoretical basis of the DOAS technique represented by the Beer-Lambert law is well studied. In contrast, scarcely investigated is the theoretical basis and validity range of the DOAS method for those cases where the contribution of the multiple scattering processes is not negligible. Our study is intended to fill this gap by means of a theoretical investigation of the applicability of the DOAS technique for the retrieval of amounts of atmospheric species from observations of the scattered solar light with a non-negligible contribution of the multiple scattering.

Starting from the expansion of the intensity logarithm in the functional Taylor series we formulate the general form of the DOAS equation. The thereby introduced variational derivative of the intensity logarithm with respect to the variation of the gaseous absorption coefficient, which is often referred to as the weighting function, is demonstrated to be closely related to the air mass factor. Employing some approximations we show that the general DOAS equation can be rewritten in the form of the weighting function (WFDOAS), the modified (MDOAS), and the standard DOAS equations. For each of these forms a specific equation for the air mass factor follows which, in general, is not suitable for other forms of the DOAS equation. Furthermore, the va-
\end{abstract}

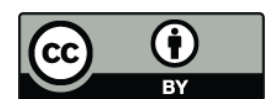

Correspondence to: V. V. Rozanov (rozanov@iup.physik.uni-bremen.de) lidity range of the standard DOAS equation is quantitatively investigated using a suggested criterion of a weak absorption.

The results presented in this study are intended to provide a basis for a better understanding of the applicability range of different forms of the DOAS equation as well as of the relationship between the air mass factor and the weighting function. To facilitate the understanding of the paper content for unexperienced reader we start our discussion considering in detail the standard DOAS technique applied to the observations of the direct solar light transmitted through the Earth's atmosphere.

\section{Introduction}

The basic idea behind the usage of the Differential Optical Absorption Spectroscopy (DOAS) to detect atmospheric constituents can be traced back to Brewer et al. (1973) and Noxon et al. (1979), who have determined $\mathrm{NO}_{2}$ concentrations from the measurements of the transmitted solar light and zenith sky scattered light. Platt and Perner (1980) applied this technique for a long path measurements of tropospheric gases using an artificial light source. The DOAS method has been further improved by Solomon et al. (1987) and later by Richter et al. (1999) to retrieve tropospheric and stratospheric constituents from ground-based zenith sky measurements. Burrows et al. (1999) have successfully exploited the DOAS technique to retrieve amounts of atmospheric trace gases from space-borne multispectral measurements of the scattered solar light in the UV-visible spectral range. Further modification of the approach has been proposed by Buchwitz et al. (2000) allowing the total columns of atmospheric species to be retrieved from the

Published by Copernicus Publications on behalf of the European Geosciences Union. 
backscattered solar radiance measured in the near-infrared spectral range by the SCanning Imaging Absorption spectroMeter for Atmospheric CHartographY (SCIAMACHY) (Bovensmann et al., 1999) in nadir viewing geometry. A comprehensive review of DOAS technique applications is presented by Platt and Stutz (2008).

Being originally developed to analyze measurements of the transmitted light, the DOAS approach is widely used for the interpretation of multispectral measurements of scattered solar light as well. The theoretical basis of this extension is, however, up to now scarcely investigated. Most critical is the extension of the DOAS technique to observation scenarios where the contribution of multiple scattering processes is significant and the gaseous absorption is non-weak (this is typical, for example, for ground-based or satellite observations in Hartley-Huggins absorption bands of ozone). This fact is illustrated, for example, in the work of Roscoe et al. (1999) who have presented the results of the Network for the Detection of Stratospheric Change inter-comparison campaign. The study has shown that vertical column densities of $\mathrm{O}_{3}$ obtained using a simple single scattering approximation to calculate the air mass factor (AMF) agree better with measurements from balloon-borne instruments than the vertical column densities derived employing a more accurate multiple scattering radiative transfer model to calculate the air mass factor (Marquard et al., 2000). This can be explained taking into account that the standard DOAS equation is solved with respect to a wavelength independent parameter which is referred to as the slant column density of an absorbing gas. However, to convert the slant column into the vertical column an additional equation is required which is not strictly defined in the framework of the standard DOAS technique. Although the expression for the air mass factor as proposed in Perliski and Solomon (1993); Sarkissian et al. (1995) provides a linear relation between the slant column and the vertical column, it can not be directly used in the framework of the standard DOAS technique because it requires the air mass factor to be constant within the selected spectral window whereas the air mass factor suggested in Perliski and Solomon (1993); Sarkissian et al. (1995) is wavelength dependent. Therefore, an additional assumption is needed to convert the slant column into the vertical column which causes an increased uncertainty of the results.

Although there are other forms of the DOAS equation that do not require the air mass factor to be wavelength independent, these are not widely used because their validity range and the relationship to the standard DOAS technique are scarcely investigated.

The main goal of this paper is to derive several forms of the DOAS equation allowing for a significant contribution of multiple scattering processes and to demonstrate that for each form a distinct expression for the air mass factor follows. This is done employing the general linear perturbation approach. The performance of different forms of the DOAS equation is investigated and shown to be different in a gen- eral case becoming, however, similar for a weakly absorbing medium. Furthermore, we introduce a quantitative criterion of a weak gaseous absorption and demonstrate that all considered forms of the DOAS equation become nearly equivalent once this criterion is satisfied.

To simplify the discussion below we do not account for the finite spectral resolution of the observing system, i.e., we do not consider the convolution of the incident radiance with the instrument slit function (so-called monochromatic treatment). Furthermore, the equations are derived considering only one absorbing species. These assumptions, however, are solely to simplify the mathematical formulations and do not affect the generality of the obtained results. Being of minor importance for theoretical investigations, the spectral convolution of the radiance is obviously important when applying the DOAS technique to real observations. An appropriate modification of the standard DOAS technique allowing for the finite spectral resolution of the observing system is presented among others by Frankenberg et al. (2005).

The layout of the paper is as follows. In Sect. 2 we introduce the standard DOAS equation considering the direct solar light transmitted through the Earth's atmosphere. Although most results presented in this section are not new, these are summarized here for the convenience of readers. Additional problems arising when applying the standard DOAS technique to multispectral measurements of the scattered solar light are discussed in Sect. 3. Several equivalent representations of the standard DOAS equation are summarized in Sect. 4. In Sect. 5 we derive the generalized form of the DOAS equation expanding the intensity in the functional Taylor series with respect to the variation of the vertical distribution of the gaseous absorption coefficient. The weighting function DOAS (WFDOAS) equation and the corresponding expressions for the air mass factor are derived in Sect. 6 employing the generalized DOAS equation formulated in Sect. 5. Several equivalent expressions for the AMF compatible to the WFDOAS equation are derived and discussed in Appendix C. In Sect. 7 we present the modified DOAS equation (MDOAS) which is obtained from the WFDOAS equation employing a specific finite-difference representation for the weighting function. The expression for the wavelength independent AMF corresponding to the standard DOAS equation is presented in Sect. 8. The relationship between different forms of the DOAS equation is discussed in Sect. 9. A quantitative criterion of a weak gaseous absorption is introduced in Sect. 10. Furthermore, it is demonstrated in this section that all considered forms of the DOAS equation become nearly equivalent once this criterion is satisfied. Other recently published approaches to derive air mass factors (Slusser et al., 1996; Rozanov et al., 1998; Stammes and Koelemeijer, 1999; Marquard et al., 2000; Palmer et al., 2001) as well as their relationship to the presented forms of the DOAS equation and associated air mass factors are discussed in Sect. 11. We leave, however, out of the consideration publications which propose improvements in the DOAS 
fit procedure affecting only the quality of spectral fit and use the standard approach to describe the amount of the target species, e.g., Pukite et al. (2009). In Sect. 12 we consider some example numerical simulations illustrating the performance of different DOAS equations being applied to the retrieval of ozone vertical columns from space-borne multispectral measurements of the scattered solar light in the UVvisible spectral range.

\section{Standard DOAS technique: transmitted light}

In this section we introduce the essential terminology commonly used in the framework of the DOAS technique and derive the DOAS equation as well as the expression for the air mass factor in the simplest case of the direct solar light transmitted through the Earth's atmosphere. Unlike a usual treatment of the standard DOAS technique as presented among others by Marquard et al. (2000) we permit spatial variations of the gaseous absorption coefficient. This allows us to get a better understanding of the assumptions needed to be done when formulating the standard DOAS equation.

The simplest way to introduce the DOAS technique is to consider ground-based measurements of the direct solar light transmitted through the Earth's atmosphere, i.e., the instrument is located at the ground and looks towards the sun. For this observation geometry the relationship between the intensity, $E_{0}(\lambda)$, at the top of atmosphere and the intensity of the transmitted solar light detected by the instrument, $E(\lambda)$, is provided by the Beer-Lambert law, see, e.g., Thomas and Stamnes (1999):

$E(\lambda)=E_{0}(\lambda) \mathrm{e}^{-t_{\mathrm{s}}(\lambda)}$.

In the framework of the DOAS technique $t_{\mathrm{S}}(\lambda)$ is referred to as the slant optical thickness and is introduced as follows:

$t_{\mathrm{s}}(\lambda)=\int_{l_{1}}^{l_{2}} e_{\lambda}(l) d l$

where $e_{\lambda}(l)$ is the extinction coefficient of the atmosphere at a wavelength $\lambda, d l$ is a path length element, and the integration is performed along the light path between the top of the atmosphere $\left(l_{1}\right)$ and the entrance slit of the instrument $\left(l_{2}\right)$. Clearly, the slant optical thickness given by Eq. (2) depends not only on the wavelength but also on the solar zenith angle. This dependence, however, will not be explicitly indicated in this section. In contrast to the slant optical thickness, the vertical optical thickness, $\tau(\lambda)$, is introduced as

$\tau(\lambda)=\int_{0}^{H} e_{\lambda}(z) d z$,

where the integration is carried out along the local vertical between the surface and the top of the atmosphere, $H$.
Let us assume the intensity of the transmitted solar light to be measured at a discrete number, $N_{\lambda}$, of wavelengths within a spectral window delimited by a minimum wavelength $\lambda_{1}$ and a maximum wavelength $\lambda_{2}$, i.e., $\lambda \in\left[\lambda_{1}, \lambda_{2}\right]$. If this spectral window contains absorption features of a certain atmospheric species, the slant optical thickness can be split into a sum

$t_{\mathrm{s}}(\lambda)=t_{\mathrm{g}}(\lambda)+t_{\mathrm{c}}(\lambda)$,

where $t_{\mathrm{g}}(\lambda)$ is attributed to the gaseous absorption and $t_{\mathrm{c}}(\lambda)$ comprises contributions due to the Rayleigh scattering and the aerosol extinction. We assume here that the atmosphere is cloud free. The slant optical thickness of the gaseous absorption is expressed as

$t_{\mathrm{g}}(\lambda)=\int_{l_{1}}^{l_{2}} \sigma_{\lambda}(l) n(l) d l$

where $\sigma_{\lambda}(l)$ is the absorption cross section $\left(\mathrm{cm}^{2} / \mathrm{molec}\right)$ and $n(l)$ is the number density $\left(\mathrm{molec} / \mathrm{cm}^{3}\right)$ of the absorbing species. In a general case, absorption cross sections of atmospheric species depend on the temperature and pressure. Logarithmizing both sides of Eq. (1) and substituting then Eqs. (4) and (5), the Beer-Lambert law is rewritten as follows:

$\ln I_{\lambda}(k)=-\int_{l_{1}}^{l_{2}} \sigma_{\lambda}(l) n(l) d l-t_{\mathrm{c}}(\lambda)$,

where $I_{\lambda}(k)=E(\lambda) / E_{0}(\lambda)$ is the sun normalized intensity corresponding to the absorption coefficient $k_{\lambda}(l)$ which is defined as $k_{\lambda}(l)=\sigma_{\lambda}(l) n(l)$. For the sake of simplicity in the following discussion $I_{\lambda}(k)$ will be referred to as the intensity.

The main purpose of measuring the transmitted solar radiation in spectral windows containing absorption bands of atmospheric species is usually to estimate the amount of these species in the atmosphere. This is supposed to be done solving Eq. (6) with respect to absorber concentrations, $n(l)$. However, as clearly seen, the latter is not the sole unknown parameter in this equation. Rather, it also contains the slant optical thickness $t_{\mathrm{c}}(\lambda)$ comprising generally unknown contributions of the Rayleigh scattering and aerosol extinction. This obstacle can be avoided taking into account that, unlike highly structured gaseous absorption signatures, the slant optical thickness $t_{\mathrm{c}}(\lambda)$ is usually a smooth function of the wavelength. Therefore, if the considered spectral window is not too wide, the wavelength dependence of $t_{\mathrm{c}}(\lambda)$ can be approximated by a polynomial of a low order, i.e.,

$t_{\mathrm{c}}(\lambda) \approx \sum_{k=0}^{N} a_{k} \lambda^{k}$

where $N$ is the order of polynomial, and $a_{k}$ are polynomial coefficients, which are unknown at this point. This assumption requires the absorption cross section $\sigma_{\lambda}(l)$ to show rapid 
variations with the wavelength. Following Platt (1994), we assume that the absorption cross section of a considered atmospheric species can be split into two components:

$\sigma_{\lambda}(l)=\sigma_{\lambda}^{c}(l)+\sigma_{\lambda}^{d}(l)$,

where, within the selected spectral window, $\sigma_{\lambda}^{c}(l)$ varies only slowly and $\sigma_{\lambda}^{d}(l)$ shows rapid variations with the wavelength. In the framework of the DOAS technique $\sigma_{\lambda}^{d}(l)$ is usually referred to as the differential absorption cross section (Platt, 1994).

Substituting $t_{\mathrm{c}}(\lambda)$ as given by Eq. (7) into Eq. (6) and neglecting the approximation error, we obtain

$\ln I_{\lambda}(k)=-\int_{l_{1}}^{l_{2}} \sigma_{\lambda}(l) n(l) d l+\sum_{k=0}^{N} a_{k} \lambda^{k}$.

This equation provides a linear relationship between the measured function, $\ln I_{\lambda}(k)$, and the function to be retrieved, $n(l)$. The contributions of other unknown parameters such as Rayleigh scattering and aerosol extinction are approximated by the polynomial. In the following discussion equations of this kind will be referred to as the DOAS equations. Clearly, the requirement of a rapidly varying with the wavelength absorption cross section is crucial for the formulation of a DOAS equation. Indeed, substituting $\sigma_{\lambda}(l)$ as given by Eq. (8) into Eq. (9) and taking into account that the integral containing product $\sigma_{\lambda}^{c}(l) n(l)$ can be approximated by a polynomial, we rewrite Eq. (9) in the following form:

$\ln I_{\lambda}(k)=-\int_{l_{1}}^{l_{2}} \sigma_{\lambda}^{d}(l) n(l) d l+\sum_{k=0}^{N} a_{k} \lambda^{k}$.

As can be seen on the right side of Eq. (10) information about the amount of absorbing species can only be obtained if $\sigma_{\lambda}^{d}(l)$ can not be approximated by a polynomial of the same order.

It is worth noticing here, that Eq. (10) can not be solved before the relationship between $d l$ and $d z$ (i.e., between path elements along the photon path and along the local vertical) is defined. Although this relationship is obvious for observations of the direct solar light transmitted through the atmosphere (see Eq. 17 below), we do not use it at this point to retain the generality of the standard DOAS technique. In the following subsections we obtain a specific form of the DOAS equation which will be referred to as the standard DOAS equation. This equation is derived starting from Eq. (9) separately for a constant (i.e., altitude independent) and for a spatially variable absorption cross section.

\subsection{Constant absorption cross section}

\subsubsection{Standard DOAS equation and DOAS fit}

In most of recent publications considering the standard DOAS technique absorption cross sections of atmospheric species are considered to be independent of the location in the atmosphere, e.g., of the altitude (Platt, 1994; Marquard et al., 2000; Platt and Stutz, 2008). Following this assumption, Eq. (9) can be rewritten as

$\ln I_{\lambda}(k)=-\sigma_{\lambda} \int_{l_{1}}^{l_{2}} n(l) d l+\sum_{k=0}^{N} a_{k} \lambda^{k}$.

In the framework of the standard DOAS technique the integral of the number density along the light path in the right hand side of Eq. (11) is used as a measure of the gaseous absorber amount and referred to as the slant column density or slant column (Marquard et al., 2000; Hönninger et al., 2004; Wagner et al., 2007), i.e.,

$S=\int_{l_{1}}^{l_{2}} n(l) d l$.

Substituting the slant column as given by Eq. (12) into Eq. (11) we obtain the governing equation of the standard DOAS technique:

$\ln I_{\lambda}(k)=-S \sigma_{\lambda}+\sum_{k=0}^{N} a_{k} \lambda^{k}$.

From the mathematical point of view Eq. (13) is a linear algebraic system consisting of $N_{\lambda}$ equations and containing $N+2$ unknown parameters, namely, $N+1$ polynomial coefficients, $a_{k}$, and the slant column $S$. These parameters can be determined by means of a least-square fit performed in a selected spectral window $\left[\lambda_{1}, \lambda_{2}\right]$. The solution of Eq. (13) represents the first step of the standard DOAS technique which is usually referred to as the DOAS fit.

We note that the usage of the slant column as a parameter to be retrieved is one of the major attributes of the standard DOAS technique that allows the inverse problem of the gaseous absorber amount retrieval to be formulated without specifying the relationship between $d l$ and $d z$. Further in the course of the discussion, any equation which similarly to Eq. (13) contains the slant column as a characteristic of the gaseous absorber amount will be referred to as the standard DOAS equation.

\subsubsection{Conversion of the slant column to the vertical column}

The slant column defined according to Eq. (12) depends not only on the absorber number density but also on the light path in the atmosphere and, hence, on the observation geometry. Therefore, when interpreting real atmospheric measurements the parameter of interest is usually the vertical column density (vertical column) of an atmospheric species rather than the slant column as resulted from the DOAS fit. The former is introduced as the integral of the absorber number density 
along the local vertical between the surface and the top of the atmosphere and is independent of the observation geometry:

$V=\int_{0}^{H} n(z) d z=\sum_{i=1}^{N_{L}} V_{i}=\sum_{i=1}^{N_{L}} \int_{z_{i-1}}^{z_{i}} n(z) d z$,

where, $V_{i}$ is the so-called partial vertical column in the $i$-th altitude layer. The transformation of the slant column obtained from the DOAS fit into the vertical column represents the second step of the standard DOAS technique. The transformation coefficient usually referred to as the enhancement factor or the air mass factor (Solomon et al., 1987; McKenzie et al., 1991) is defined as follows:

$A=\frac{S}{V}$.

Substituting the slant column and the vertical column as given by Eqs. (12) and (14), respectively, Eq. (15) is rewritten as

$$
A=\frac{\int_{l_{1}}^{l_{2}} n(l) d l}{\int_{0}^{H} n(z) d z} .
$$

Thus, to complete the second step of the DOAS retrieval procedure the air mass factor needs to be calculated. As seen from Eq. (16) this requires a relationship between $d l$ and $d z$ to be specified which was not needed before (i.e., at the DOAS fit step). For observations of the direct solar light transmitted through the atmosphere this relationship is obvious. Indeed, assuming for a simplicity reason the atmosphere to be plane-parallel and the solar zenith angle at the top of atmosphere to be $\vartheta_{0}$, we have:

$d l=\frac{1}{\mu_{0}} d z$,

where $\mu_{0}=\cos \vartheta_{0}$. Substituting $d l$ as given by Eq. (17) into Eq. (16) we obtain

$A=\frac{1}{\mu_{0}}$.

Thus, the slant column, $S$, obtained from the DOAS fit is transformed into the vertical column as follows:

$V=\mu_{0} S$.

\subsection{Spatially variable absorption cross section}

\subsubsection{Standard DOAS equation and DOAS fit}

To derive the DOAS equation similar to Eq. (13) for a spatially variable absorption cross section an additional assumption needs to be introduced, namely, the number density profile, $n(l)$, is considered to be obtained by a scaling of a known profile, $\bar{n}(l)$, within the entire atmosphere, i.e.,

$n(l)=C \bar{n}(l)$, where $C$ is a constant to be determined and $\bar{n}(l)$ is the socalled a priori number density profile. Substituting now $n(l)$ as given by Eq. (20) into Eq. (9) we obtain

$\ln I_{\lambda}(k)=-S \frac{1}{\bar{S}} \int_{l_{1}}^{l_{2}} \sigma_{\lambda}(l) \bar{n}(l) d l+\sum_{k=0}^{N} a_{k} \lambda^{k}$,

where we have taken into account that according to Eq. (12) a scaling of the number density profile results in the same scaling of the associated slant column, i.e., $S=C \bar{S}$, where $\bar{S}$ is the slant column corresponding to the number density profile $\bar{n}(l)$. Introducing further the slant path weighted absorption cross section as

$\tilde{\sigma}_{\lambda}=\frac{1}{\bar{S}} \int_{l_{1}}^{l_{2}} \sigma_{\lambda}(l) \bar{n}(l) d l$,

and substituting it into Eq. (21) we obtain

$\ln I_{\lambda}(k)=-S \tilde{\sigma}_{\lambda}+\sum_{k=0}^{N} a_{k} \lambda^{k}$

Although this equation is very similar to the standard DOAS equation as given by Eq. (13) it can not be directly used to obtain the slant column, $S$, employing the DOAS fit. This is because an integration over the light path needs to be done when calculating the slant path weighted cross section, $\tilde{\sigma}_{\lambda}$, according to Eq. (22) that in turn requires an explicit relationship between $d l$ and $d z$ to be specified already at the DOAS fit step. To avoid this, we have to assume that the slant path weighted absorption cross section is the same as the vertical path weighted cross section defined by

$\hat{\sigma}_{\lambda}=\frac{1}{\bar{V}} \int_{0}^{H} \sigma_{\lambda}(z) \bar{n}(z) d z=\frac{\bar{\tau}(\lambda)}{\bar{V}}$.

Here, the vertical optical thickness of the trace gas absorption, $\bar{\tau}(\lambda)$, is introduced according to Eq. (3) for $e_{\lambda}(z)=$ $\sigma_{\lambda}(z) \bar{n}(z)$. This assumption allows us to estimate the slant column of the absorbing species applying the DOAS fit procedure to Eq. (23) in the same manner as for Eq. (13).

\subsubsection{Conversion of the slant column to the vertical column}

For the slant column obtained employing the DOAS fit according to Eq. (23) the transformation into the vertical column is done in exactly the same manner as in the case of a constant absorption cross section, i.e., as given by Eq. (19).

Summing up all results obtained above, the standard DOAS equation system suitable for the interpretation of observations of the direct solar light transmitted through the atmosphere (in a plane-parallel approximation) is written as follows:

$\ln I_{\lambda}(k)=-S \hat{\sigma}_{\lambda}+\sum_{k=0}^{N} a_{k} \lambda^{k}$ 
Table 1. Assumptions employed to derive the DOAS equations (13) and (25) suitable for the retrieval of gaseous absorber amounts from observations of the direct solar light transmitted through the atmosphere.

\begin{tabular}{|c|c|c|c|}
\hline \multirow[t]{2}{*}{ Num } & \multirow[t]{2}{*}{ Assumptions } & \multicolumn{2}{|c|}{ DOAS equation } \\
\hline & & $\begin{array}{l}\text { constant } \\
\text { cross } \\
\text { section }\end{array}$ & $\begin{array}{c}\text { spatially } \\
\text { variable cross } \\
\text { section }\end{array}$ \\
\hline 1 & $\begin{array}{l}\text { the gaseous absorption cross section has to contain a component } \\
\text { rapidly varying with the wavelength }\end{array}$ & + & + \\
\hline 2 & $\begin{array}{l}\text { within the considered spectral window the wavelength dependence } \\
\text { of the slant optical thickness due to the Rayleigh scattering and the } \\
\text { aerosol extinction can be approximated by a polynomial of a low } \\
\text { order }\end{array}$ & + & + \\
\hline 3 & $\begin{array}{l}\text { the scaling approximation of the absorber vertical profile, see } \\
\text { Eq. (20) }\end{array}$ & not applied & + \\
\hline
\end{tabular}

$A=\frac{1}{\mu_{0}}$,

$V=\frac{S}{A}$

where $\hat{\sigma}_{\lambda}$ is the vertical path weighted absorption cross section as given by Eq. (24). The assumptions used to derive these equations are summarized in Table 1 . Note that for observations of the direct solar light transmitted through a plane-parallel atmosphere the slant path weighted cross section, $\tilde{\sigma}_{\lambda}$, as given by Eq. (22), is exactly the same as the vertical path weighted cross section, $\hat{\sigma}_{\lambda}$, given by Eq. (24). This is the reason why Table 1 does not contain the assumption of $\tilde{\sigma}_{\lambda}=\hat{\sigma}_{\lambda}$.

Concluding, let us summarize main definitions related to the DOAS technique which will be used in the following discussion:

- DOAS equation is an equation providing a linear relationship between the intensity logarithm and a gaseous absorber amount where the contribution of other unknown atmospheric parameters is approximated by a low order polynomial.

- Slant column is the integral absorber amount along the light path between the light source and the instrument.

- Standard DOAS equation is an equation providing a linear relationship between the intensity logarithm and the slant column of a gaseous absorber where the contribution of other unknown atmospheric parameters is approximated by a low order polynomial.

- Vertical column is the integral absorber amount along the vertical path between the bottom and the top of the atmosphere.
- DOAS fit denotes solving the standard DOAS equation with respect to the slant column.

- Air mass factor is the ratio of the slant column to the vertical column.

- Standard DOAS technique comprises the DOAS fit followed by the conversion of the slant column into the vertical column using an appropriate air mass factor.

\section{Standard DOAS technique: scattered light}

In this section we discuss some additional problems arising when employing the standard DOAS technique to retrieve amounts of atmospheric species from multispectral measurements of the solar light scattered in the Earth's atmosphere and reflected from the surface.

\subsection{Standard DOAS equation and DOAS fit}

As discussed by Platt and Stutz (2008) in their comprehensive review of standard DOAS technique applications, the standard DOAS equation as given by Eq. (25) is also employed to retrieve amounts of atmospheric species from multispectral measurements of the scattered solar light with a non-negligible contribution of the multiple scattering. However, as the multiple scattering causes the light path to depend on the scattering properties of the atmosphere, i.e., on such processes as Rayleigh scattering, aerosol extinction, surface reflection, and so on, the slant column defined by Eq. (12) as the integral over the light path becomes wavelength dependent. Thus, when employing the standard DOAS equation to retrieve the amount of a gaseous absorber from observations of this kind, the wavelength dependence of the slant column is neglected. Moreover, as discussed by Stammes and Koelemeijer (1999), for a spatially variable absorption 
cross section one has to assume the slant path weighted absorption cross section as given by Eq. (22) to be the same as the vertical path weighted cross section as given by Eq. (24). This, however, is not necessarily the case for observations of scattered solar light.

For further considerations let us rewrite the standard DOAS equation as given by Eq. (25) introducing the subscript " $j$ " which emphasizes the dependence of the intensity logarithm and the slant column on the observation conditions:

$\ln I_{\lambda, j}(k)=-S_{j} \hat{\sigma}_{\lambda}+\sum_{k=0}^{N} a_{k} \lambda^{k}$.

Although the polynomial coefficients, $a_{k}$, depend on the observation conditions as well, this dependence will not be indicated hereinafter. The set of the observation conditions needed to perform the retrieval is usually determined by the measurement technique. For example, for ground-based zenith sky observations of the scattered solar light (Noxon et al., 1979; McKenzie and Johnston, 1982; Solomon et al., 1987) the slant columns are usually obtained at different solar zenith angles or in different spectral windows (Postylyakov et al., 2006) whereas Multiple AXis Differential Optical Absorption Spectroscopy (MAX-DOAS) observations are performed at similar illumination conditions and various viewing angles (see e.g. Hönninger et al., 2004; Bruns et al., 2004, 2006). The set of slant columns obtained at different observation conditions is often used to retrieve vertical distributions of the absorber number density, e.g., Hendrick et al. (2004); Haley et al. (2004).

It is worth noticing here that it was Harrison (1979) who introduced the standard DOAS equation in a form very similar to Eq. (28) and employed it for the first time to retrieve $\mathrm{NO}_{2}$ and $\mathrm{O}_{3}$ slant columns from zenith-sky observations of the scattered solar light. Subsequently this equation was employed by McKenzie and Johnston (1982) to perform the same kind of retrievals.

\subsection{Conversion of the slant column to the vertical column}

As mentioned above, a set of slant columns obtained from the DOAS fit for different observation conditions contains information about the vertical distribution of the considered atmospheric species. In the framework of the DOAS technique this set of slant columns is commonly used to retrieve the partial vertical columns, $V_{i}$, of the gaseous absorber within atmospheric layers, see Eq. (14). According to Solomon et al. (1987); McKenzie et al. (1991), the relationship between the slant column and partial vertical columns is given by

$S_{j}=\sum_{i=1}^{N_{L}} A_{i, j} V_{i}, j=1,2, \ldots, N_{s}$,

where $S_{j}$ is the slant column corresponding to $j$-th observation, $N_{S}$ is the number of observation conditions, $N_{L}$ is the number of atmospheric layers, and coefficients $A_{i, j}$ are usually referred to as the box-air mass factors (box-AMFs). In the framework of the standard DOAS technique the boxAMF is defined as the ratio of the partial slant column to the partial vertical column (Hönninger et al., 2004; Wagner et al., 2007):

$A_{i, j}=\frac{S_{i, j}}{V_{i}}=\frac{\int_{l_{i-1}}^{l_{i}} n\left(l_{j}\right) d l_{j}}{\int_{z_{i-1}}^{z_{i}} n(z) d z}$.

where $d l_{j}$ and $d z$ are the actual light path and the vertical path, respectively. The box-AMF is considered as a measure of enhancement of the light path within an atmospheric layer with respect to the vertical path in this layer due to the observation geometry and scattering processes. Clearly, the definition of the box-AMF given by Eq. (30) is in line with the definition of the air mass factor for the whole atmosphere as given by Eq. (15). Following Wagner et al. (2007) the latter will be referred to below as the total AMF. As shown in Wagner et al. (2007) the total AMF can be easily obtained from the box-AMFs $\left(A_{i, j}\right)$ and the vertical profile of the respective trace gas as

$A_{j}=\frac{\sum_{i=1}^{N_{L}} A_{i, j} V_{i}}{\sum_{i=1}^{N_{L}} V_{i}}=\frac{1}{V} \sum_{i=1}^{N_{L}} A_{i, j} V_{i}$.

Unlike observations of the direct solar light transmitted through the atmosphere no simple equation for the air mass factor similar to Eq. (26) can be obtained when observing the scattered solar light with a non-negligible contribution of the multiple scattering processes. Furthermore, the AMF can not be calculated according to its definition as given by Eq. (30) because it requires an integration of a gaseous absorber number density along the actual light path which is generally unknown because of a significant contribution of the multiple scattering. In the past decades, several different expressions to calculate the air mass factor accounting for multiple scattering effects have been suggested in the literature. Let us consider here the most commonly used method proposed by Perliski and Solomon (1993) and Sarkissian et al. (1995),

$A_{i, j}(\lambda)=\frac{\ln I_{\lambda, j}(0)-\ln I_{\lambda, j}\left(\bar{k}_{i}\right)}{\sigma_{\lambda} \bar{V}_{i}}$,

whereas other expressions are discussed in Sect. 11 in details. Intensities of the diffuse light, $I_{\lambda, j}\left(\bar{k}_{i}\right)$ and $I_{\lambda, j}(0)$, in Eq. (32) are calculated using an appropriate radiative transfer model. The former is computed assuming the absorber number density of $\bar{n}_{i}(z)$ in the $i$-th altitude layer and the zero amount everywhere else whereas the latter is obtained for the zero amount of the absorber in the whole atmosphere. Here, $\bar{n}(z)$ is an initial guess (a priori) vertical distribution of the 
absorbing species and $\bar{V}_{i}$ are its partial vertical columns as defined by Eq. (14). The motivation to introduce the AMF in this manner can be easily understood if, following Perliski and Solomon (1993), we rewrite Eq. (32) in the form of the Beer-Lambert law:

$I_{\lambda, j}\left(\bar{k}_{i}\right)=I_{\lambda, j}(0) e^{-\sigma_{\lambda} \bar{V}_{i} A_{i, j}(\lambda)}$.

Therewith $A_{i, j}(\lambda)$ is introduced as a factor accounting for the effective optical path enhancement (both geometrical and due to multiple scattering processes) for an atmosphere containing one absorbing layer. It is worth noticing here that the AMF given by Eq. (32) is wavelength dependent as, in contrast to observations of the direct solar light transmitted through the atmosphere (see Eq. 26), it is determined not only by the observational geometry but also by atmospheric properties such as the Rayleigh scattering, aerosol extinction, amount of gaseous absorber, and surface reflection. This behavior is not surprising because these atmospheric parameters govern scattering processes in the atmosphere and, therefore, the photon path lengths.

Summarizing results obtained in this section, the mathematical representation of the standard DOAS technique suitable to interpret observations of the scattered solar light with a non-negligible contribution of the multiple scattering can be formulated as follows:

$$
\begin{aligned}
& \ln I_{\lambda, j}(k)=-S_{j} \hat{\sigma}_{\lambda}+\sum_{k=0}^{N} a_{k} \lambda^{k}, j=1,2, \ldots, N_{S}, \\
& A_{i, j}(\lambda)=\frac{\ln I_{\lambda, j}(0)-\ln I_{\lambda, j}\left(\bar{k}_{i}\right)}{\hat{\sigma}_{\lambda} \bar{V}_{i}}, i=1,2, \ldots, N_{L}, \\
& A_{i, j}(\lambda) \longrightarrow A_{i, j}, \\
& S_{j}=\sum_{i=1}^{N_{L}} A_{i, j} V_{i} .
\end{aligned}
$$

Here, $A_{i, j}$ is a representative value for the wavelength independent AMF which is needed to transform the slant column into the vertical column according to Eq. (37). However, in contrast to the DOAS equation system obtained for observations of the direct solar light as given by Eqs. (25-27), the DOAS equation system given by Eqs. (34-37) is underdetermined because the rule to transform the wavelength dependent air mass factor, $A_{i, j}(\lambda)$, into a wavelength independent value, $A_{i, j}$, is not defined. This missing mapping rule emphasized by the symbolic link $A_{i, j}(\lambda) \longrightarrow A_{i, j}$ is the main weakness of the standard DOAS technique in application to the retrieval of trace gas amounts from observations of the scattered solar light. In practical applications of the technique additional approximations are used to define the missing mapping rule. For example, in a spectral region where the atmosphere can be considered to be optically thin a reasonable approximation for the wavelength independent air mass factor, $A_{i, j}$, is provided by the mean value of $A_{i, j}(\lambda)$ or by its value at the central wavelength of the considered spectral interval. For a strong gaseous absorption, however, the air mass factor, $A_{i, j}(\lambda)$, shows a significant wavelength dependence and a better approximation for the constant AMF is provided by the smallest value of $A_{i, j}(\lambda)$ in the considered spectral window, see Burrows et al. (1999) for further details.

We note that the problem of the missing mapping rule outlined above arises because the slant column is assumed to be wavelength independent when formulating the standard DOAS equation. Indeed, substituting the slant column, $S_{j}$, as given by Eq. (37) into Eq. (34) and accounting for the wavelength dependence of the box-AMF, the following expression is obtained:

$\ln I_{\lambda, j}(k)=-\sum_{i=1}^{N_{L}} V_{i} A_{i, j}(\lambda) \hat{\sigma}_{\lambda}+\sum_{k=0}^{N} a_{k} \lambda^{k}$.

This equation can be considered as a DOAS equation containing partial vertical columns, $V_{i}$, instead of the slant column, $S_{j}$, as parameters to be retrieved. However, this is not the standard DOAS equation any more. Rather, it represents so-called modified DOAS equation originally proposed by Diebel et al. (1994) and Fish et al. (1995) to improve the DOAS retrieval. The modified DOAS equation is discussed in Sect. 7 in details.

Concluding our discussion we summarize main features and assumptions associated to the standard DOAS technique in application to the retrieval of amounts of atmospheric species from multispectral measurements of the scattered solar light.

The standard DOAS technique is a specific two-step approach capable to retrieve amounts of absorbing species. At the first step usually referred to as the DOAS fit a least square fit in the spectral domain is performed to obtain the so-called slant column. The main advantage at this step is that no information about the actual light path in the atmosphere is required to retrieve the slant column. At the second step the slant column is transformed into the vertical column or into the partial vertical columns. To do this an appropriate equation for the air mass factor or box-AMF needs to be formulated.

Main assumptions associated to the standard DOAS technique are the following (the assumptions are required to hold in the considered spectral interval only):

- the absorption cross section of the retrieved species has to contain a component rapidly varying with the wavelength;

- the wavelength dependence of the non-gaseous (Rayleigh scattering and aerosol extinction) slant optical thickness can be approximated by a polynomial of a low order;

- for a spatially variable absorption cross section the slant path weighted cross section as given by Eq. (22) is 
assumed to be equal to the vertical path weighted cross section as given by Eq. (24);

- the slant column is considered to be independent of the wavelength;

- a rule to map the wavelength dependent AMF to a representative wavelength independent value has to be established.

\section{Equivalent forms of the standard DOAS equation}

In this section we discuss other equivalent representations of the standard DOAS equation as given by Eq. (34) which can be found in the literature. For a simplicity reason, let us first rewrite Eq. (34) introducing $L_{\lambda, j}(k)=\ln I_{\lambda, j}(k)$ :

$L_{\lambda, j}(k)=-S_{j} \hat{\sigma}_{\lambda}+\sum_{k=0}^{N} a_{k} \lambda^{k}$

Other representations of the standard DOAS equation are obtained considering the absorption cross section as the following sum (Platt, 1994; Richter, 1997):

$\hat{\sigma}_{\lambda}=\hat{\sigma}_{\lambda}^{c}+\hat{\sigma}_{\lambda}^{d}$,

where $\hat{\sigma}_{\lambda}^{c}$ describes a general "slope" of the absorption cross section, whereas $\hat{\sigma}_{\lambda}^{d}$ shows rapid variations with the wavelength. Substituting $\hat{\sigma}_{\lambda}$ as given by Eq. (40) into Eq. (39) and taking into account that $\hat{\sigma}_{\lambda}^{c}$ can be approximated by a low order polynomial, we rewrite the standard DOAS equation given by Eq. (39) in the following equivalent form:

$L_{\lambda, j}(k)=-S_{j} \hat{\sigma}_{\lambda}^{d}+\sum_{k=0}^{N} a_{k} \lambda^{k}$

Similar to Eq. (40) for the absorption cross section, the intensity logarithm in the left hand side of Eq. (41) can be also represented as a sum of two components, respectively varying slowly and rapidly with the wavelength:

$L_{\lambda, j}(k)=L_{\lambda, j}^{c}(k)+L_{\lambda, j}^{d}(k)$.

As, according to Eq. (1), $-L_{\lambda, j}(k)$ is equal to the slant optical thickness, $t_{\mathrm{S}}(\lambda)$, the term $-L_{\lambda, j}^{d}(k)$ is often referred to as the differential slant optical thickness. Taking into account that $L_{\lambda, j}^{c}(k)$ can be approximated by a low order polynomial as well, we rewrite Eq. (41) in the following equivalent form:

$L_{\lambda, j}^{d}(k)=-S_{j} \hat{\sigma}_{\lambda}^{d}+\sum_{k=0}^{N} a_{k} \lambda^{k}$

where the polynomial contains among others the contributions of $S_{j} \hat{\sigma}_{\lambda}^{c}$ and $L_{\lambda, j}^{c}(k)$. Although not explicitely indicated, the polynomial coefficients in Eqs. (39), (41), and (43) are different.
In addition, the form of the standard DOAS equation depends on the approach used to separate general slopes of the absorption cross section and slant optical thickness, $\hat{\sigma}_{\lambda}^{c}$ and $L_{\lambda, j}^{c}(k)$, respectively. In fact, the separation technique is not yet strictly defined at this point. A commonly used approach to obtain the differential absorption cross section and differential slant optical thickness is subtracting appropriate polynomials from $\hat{\sigma}_{\lambda}$ and $L_{\lambda, j}(k)$ before the DOAS fit is performed, i.e.,

$\hat{\sigma}_{\lambda}^{d}=\hat{\sigma}_{\lambda}-\sum_{k=0}^{N} \beta_{k} \lambda^{k}, L_{\lambda, j}^{d}(k)=L_{\lambda, j}(k)-\sum_{k=0}^{N} \gamma_{k} \lambda^{k}$,

where polynomial coefficients $\beta_{k}$ and $\gamma_{k}$ are obtained minimizing the following quadratic forms:

$\left|\hat{\sigma}_{\lambda}-\sum_{k=0}^{N} \beta_{k} \lambda^{k}\right|^{2} \rightarrow \min$,

$\left|L_{\lambda, j}(k)-\sum_{k=0}^{N} \gamma_{k} \lambda^{k}\right|^{2} \rightarrow \min$.

Using this technique another specific form of the standard DOAS equation is obtained:

$L_{\lambda, j}^{d}(k)=-S_{j} \hat{\sigma}_{\lambda}^{d}$

A proof of the equivalence of Eqs. (39) and (46) is given in Appendix A.

In the following discussion, the standard DOAS equation in the form given by Eq. (34) is used.

\section{Linearization technique and generalized DOAS equation}

From the mathematical point of view the standard DOAS equation as given by Eq. (34) is a linear relationship between the intensity logarithm and the absorber slant column. For the direct solar light transmitted through the atmosphere this linear relationship is a consequence of the Beer-Lambert law written in the logarithmic form. To obtain such a linear relationship for observations of the scattered solar light accounting for the multiple scattering contribution, a linearization technique needs to be employed. In the discussion below this is done using the expansion of the intensity logarithm in the functional Taylor series.

\subsection{Linearized forward model}

For an arbitrary but differentiable function $f(x)$ a linear relationship between a variation of this function and a variation of its argument can be obtained employing the Taylor series expansion. For any $x$ around the linearization point $\bar{x}$, the Taylor series is written as

$f(x)=f(\bar{x})+\frac{d f}{d x} \Delta x$, 
where $\Delta x=x-\bar{x}$, and $d f / d x$ is the derivative of the function $f(x)$ with respect to its argument calculated at $x=\bar{x}$. Here, we have restricted our consideration with the terms linear with respect to $\Delta x$ and neglected the linearization error. It is worth noticing here that such linear relationships are often used in atmospheric remote sensing inverse problems (see e.g., Deepak, 1977; Rodgers, 2000). Unfortunately, this expansion can not be directly applied to the intensity as a function of the vertical column because, in a vertically inhomogeneous atmosphere, the intensity depends on the vertical profile of the absorption coefficient,

$k_{\lambda}(z)=\sigma_{\lambda}(z) n(z)$,

and, hence, on the absorber number density profile, $n(z)$, rather than on its vertical column. Indeed, even in the simplest case of the direct solar light transmitted through a planeparallel atmosphere the Beer-Lambert law as given by Eq. (6) results in

$\ln I_{\lambda}(k)=-\frac{1}{\mu_{0}} \int_{0}^{H} k_{\lambda}(z) d z-t_{c}(\lambda)$,

where we have taken into account that $d l=d z / \mu_{0}$, see Eq. (17). As formulated in Eq. (49), the intensity logarithm at each wavelength $\lambda$ is a functional of the absorption coefficient $k_{\lambda}(z)$. In other words, it is a function that takes a function as its argument and returns a scalar, see Hazewinkel (2002) for details. Therefore, a linear relationship between the observed intensity (or its logarithm) and the vertical column of an absorbing species can not be obtained without additional assumptions.

Regarding the intensity as a functional of the absorption coefficient profile, $k_{\lambda}(z)$, a linear relationship between the intensity logarithm and $k_{\lambda}(z)$ similar to Eq. (49) can also be obtained in a general case without specifying the observation geometry. This is done employing the expansion of the intensity logarithm in the functional Taylor series as follows:

$\ln I_{\lambda}(k)=\ln I_{\lambda}(\bar{k})+\int_{0}^{H} \frac{\delta \ln I_{\lambda}}{\delta k_{\lambda}(z)} \delta k_{\lambda}(z) d z+\varepsilon_{\operatorname{lin}}(\lambda)$.

Here, $\bar{k}_{\lambda}(z)$ and $k_{\lambda}(z)=\bar{k}_{\lambda}(z)+\delta k_{\lambda}(z)$ are the initial guess (i.e., the linearization "point") and the perturbed vertical profiles of the trace gas absorption coefficient, $I_{\lambda}(\bar{k})$ and $I_{\lambda}(k)$ are the corresponding intensities, $\varepsilon_{\operatorname{lin}}(\lambda)$ is the linearization error containing the contribution of higher order terms with respect to $\delta k_{\lambda}(z)$, and

$$
\frac{\delta \ln I_{\lambda}}{\delta k_{\lambda}(z)}=\lim _{\Delta z \rightarrow 0} \frac{\ln I_{\lambda}\left(k_{\lambda}\right)-\ln I_{\lambda}\left(\bar{k}_{\lambda}\right)}{\int_{(\Delta z)} \delta k_{\lambda}\left(z^{\prime}\right) d z^{\prime}}
$$

is the variational derivative of $\ln I_{\lambda}$ with respect to the absorption coefficient $k_{\lambda}(z)$ calculated at the initial guess profile, $\bar{k}_{\lambda}(z)$. In the framework of retrieval techniques this variational derivative is often referred to as the weighting function (WF), see, e.g., Rozanov (2006) for details. The integration in the denominator of Eq. (51) is carried out over a vertical range $\Delta z$ around an altitude $z$ and the perturbation of the absorption coefficient vertical profile, $\delta k_{\lambda}\left(z^{\prime}\right)$, is considered to be zero everywhere but in the $\Delta z$ altitude range. The latter approaches zero when calculating the limit. A comprehensive discussion of the mathematical aspects related to functionals and variational derivatives is presented among others by Volterra (1959). The perturbation of the gaseous absorption coefficient, $\delta k_{\lambda}(z)$, introduced in Eq. (50) can be caused by a variation either of the trace gas number density or of its absorption cross section. The origin of the absorption cross section variation is unimportant at this point. Thus,

$\delta k_{\lambda}(z)=\bar{\sigma}_{\lambda}(z) \delta n(z)+\delta \sigma_{\lambda}(z) \bar{n}(z)$,

where $\bar{n}(z)$ and $\bar{\sigma}_{\lambda}(z)$ are initial guesses for number density profile and absorption cross section, respectively, $\delta n(z)=$ $n(z)-\bar{n}(z)$, and $\delta \sigma_{\lambda}(z)=\sigma_{\lambda}(z)-\bar{\sigma}_{\lambda}(z)$. The initial guess number density profile is also often referred to as a priori profile.

It is worth noticing here that an expansion similar to Eq. (50) can be also written for the intensity itself rather than for its logarithm. However, as discussed, e.g., by Rozanov and Kokhanovsky (2008), the impact of the linearization error is significantly reduced when applying the linear approximation to the logarithm of the intensity. This was the reason to use $\ln I_{\lambda}$ rather than $I_{\lambda}$ in the retrieval of number density vertical profiles (Hoogen et al., 1999) and total columns (Klenk et al., 1982) of ozone from the space borne observations of the backscattered solar radiation.

There are only a few simplest cases in which weighting functions can be obtained according to their definition as given by Eq. (51). For example, it is straightforward if the intensity obeys the Beer-Lambert law for a plane-parallel medium. Indeed, substituting $\ln I_{\lambda}(k)$ and $\ln I_{\lambda}(\bar{k})$ as given by Eq. (49) in the numerator of Eq. (51) we obtain:

$$
\frac{\delta \ln I_{\lambda}}{\delta k_{\lambda}(z)}=\lim _{\Delta z \rightarrow 0} \frac{-\mu_{0}^{-1} \int_{0}^{H} \delta k_{\lambda}\left(z^{\prime}\right) d z^{\prime}}{\int_{(\Delta z)} \delta k_{\lambda}\left(z^{\prime}\right) d z^{\prime}} .
$$

Taking into account that, according to the definition of the variational derivative, the perturbation $\delta k_{\lambda}\left(z^{\prime}\right)$ is non-zero only within the vertical range $\Delta z$ around an altitude $z$, the integration range in the numerator of Eq. (53) can be reduced to the interval $\Delta z$ that leads to

$$
\frac{\delta \ln I_{\lambda}}{\delta k_{\lambda}(z)}=-\frac{1}{\mu_{0}} \lim _{\Delta z \rightarrow 0} \frac{\int_{(\Delta z)} \delta k_{\lambda}\left(z^{\prime}\right) d z^{\prime}}{\int_{(\Delta z)} \delta k_{\lambda}\left(z^{\prime}\right) d z^{\prime}}=-\frac{1}{\mu_{0}} .
$$


Comparing this expression to Eq. (18) for the air mass factor obtained for observations of the direct solar light transmitted through the atmosphere, we can state that

$A=\left|\frac{\delta \ln I_{\lambda}}{\delta k_{\lambda}(z)}\right|$,

i.e., the absolute value of the variational derivative of the intensity logarithm with respect to the gaseous absorption coefficient is equal to the air mass factor.

For most of practically important inverse problems the weighting functions can be obtained employing a very efficient technique of the joint solution of the linearized forward and adjoint radiative transfer equations. This approach and other techniques to calculate weighting functions accounting for the multiple scattering contribution are discussed in Rozanov and Rozanov (2007). The most straightforward approach is the numerical perturbation technique that uses a finite-difference approximation instead of the exact representation given by Eq. (51). The weighting function at a discrete altitude level $z_{i}$ is obtained then as follows:

$\frac{\delta \ln I_{\lambda}}{\delta k_{\lambda}\left(z_{i}\right)} \approx \frac{\ln I_{\lambda}\left(\bar{k}_{\lambda}\left(z_{i}\right)+\Delta k_{\lambda}\left(z_{i}\right)\right)-\ln I_{\lambda}\left(\bar{k}_{\lambda}\left(z_{i}\right)\right)}{\Delta k_{\lambda}\left(z_{i}\right)}$,

where $\Delta k_{\lambda}\left(z_{i}\right)$ is the variation of the absorption coefficient at the altitude level $z_{i}$ and intensities $I_{\lambda}\left(\bar{k}_{\lambda}\left(z_{i}\right)+\Delta k_{\lambda}\left(z_{i}\right)\right)$ and $I_{\lambda}\left(\bar{k}_{\lambda}\left(z_{i}\right)\right)$ are the solutions of the radiative transfer equation for the corresponding profiles of the absorption coefficient. The weighting function for the entire atmosphere is obtained using Eq. (56) at each level of the altitude grid.

Introducing the absorption coefficient weighting function as

$w_{\bar{k}}(\lambda, z)=\left.\frac{\delta \ln I_{\lambda}}{\delta k_{\lambda}(z)}\right|_{\bar{k}_{\lambda}(z)}$,

where the subscript $\bar{k}$ indicates that the weighting function is to be calculated at the initial guess profile $\bar{k}_{\lambda}(z)$, Eq. (50) can be rewritten as follows:

$\ln I_{\lambda}(k)=\ln I_{\lambda}(\bar{k})+\int_{0}^{H} w_{\bar{k}}(\lambda, z) \delta k_{\lambda}(z) d z+\varepsilon_{\operatorname{lin}}(\lambda)$.

Taking into account that a variation of the intensity can be caused by variations of other absorbing gaseous components as well as atmospheric and surface parameters such as Rayleigh scattering and aerosol extinction coefficients, surface albedo, and so on, Eq. (58) has to be extended as follows:

$\ln I_{\lambda}(p)=\ln I_{\lambda}(\bar{p})+\sum_{m=1}^{N_{\mathrm{P}}} \int_{0}^{H} w_{\bar{p}_{m}}(\lambda, z) \delta p_{m}(z) d z$.

Here, $N_{\mathrm{P}}$ is the full number of atmospheric parameters which can cause a variation of the intensity, $\delta p_{m}(z)$ are absolute variations of the atmospheric parameters, and $w_{\bar{p}_{m}}(\lambda, z)$ are the corresponding weighting functions. The last term in the right hand side of Eq. (58) denoting the linearization error is omitted here. It is worth noticing that the linearization error depends on the magnitude of $\delta p_{m}(z)$, i.e., on how strong the atmospheric parameters vary. If a perturbed parameter value is in a close neighborhood of the initial guess value, $\bar{p}_{m}(z)$, then the intensity logarithm can be considered to be nearly linear with respect to the variation $\delta p_{m}(z)$ and the linearization error is negligible. In the course of our discussion below, this assumption will be referred to as the local linearity.

Equation (59) provides a linear relationship between the intensity logarithm and variations of atmospheric parameters such as vertical profile of the gaseous absorption coefficient, Rayleigh scattering and aerosol extinction coefficients, as well as surface albedo. Thus, Eq. (59) represents a linearized forward model for the intensity logarithm. Analytical expressions for weighting functions of various atmospheric and surface parameters can be found, e.g., in Rozanov (2006); Rozanov et al. (2007).

\subsection{Generalized DOAS equation}

Restricting our consideration, similarly to the previous discussion, to only one absorbing species, the linearized forward model given by Eq. (59) is rewritten in the following form:

$\ln I_{\lambda}(k)=\ln I_{\lambda}(\bar{k})+\int_{0}^{H} w_{\bar{k}}(\lambda, z) \delta k_{\lambda}(z) d z+\delta t_{p}(\lambda)$.

Here, the second term in the right hand side of the equation describes the variation of the intensity logarithm caused by a variation of the trace gas absorption coefficient and the third term comprises contributions of all other atmospheric parameters.

Clearly, the weighting function of the absorption coefficient, $w_{\bar{k}}(\lambda, z)$, shows the same spectral features as the absorption cross section of the atmospheric species. Despite the fact that the Rayleigh scattering and aerosol extinction coefficients as well as the surface albedo have only a smooth dependence on the wavelength, their weighting functions show the spectral features associated to the gaseous absorption as well. This is because variations of these parameters influence the scattering and reflection processes which determine the light path in the atmosphere and, therefore, affect the absorption of the solar light by atmospheric trace gases. Nevertheless, if the gaseous absorption in the considered spectral range is weak enough (a quantitative characterization is provided in Sect. 10) or the contribution of the scattering processes is not too large, the wavelength dependence of corresponing weighting functions can be approximated by a 

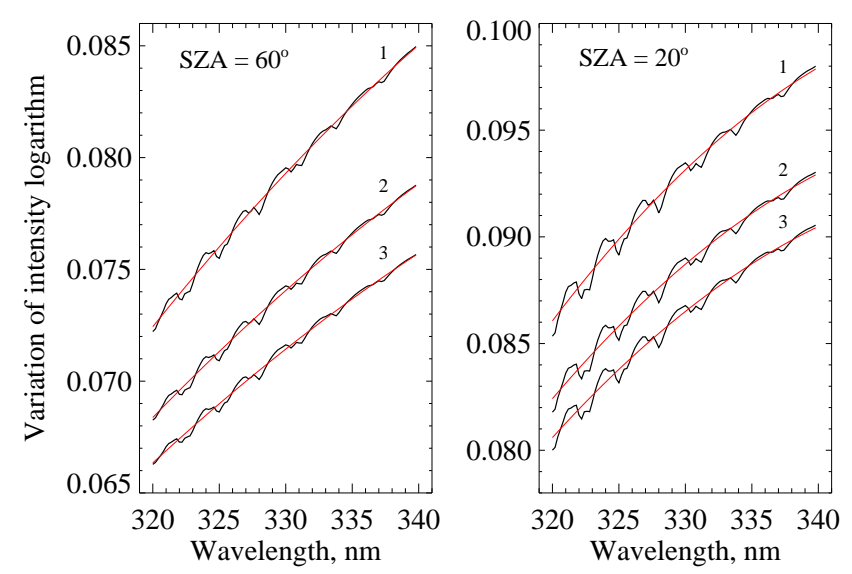

Fig. 1. Approximation of $\delta t_{p}(\lambda)$ (black) by a second order polynomial (red). The results are obtained for the Rayleigh scattering coefficient increased by $10 \%$, surface albedo increased by $10 \%$, and aerosol extinction coefficient increased by 25,15 , and $10 \%$ (marked in plot by 1,2 , and 3 , respectively).

polynomial. This means that $\delta t_{p}(\lambda)$ in the right hand side of Eq. (60) can be represented as:

$\delta t_{p}(\lambda) \approx \sum_{k=0}^{N} a_{k} \lambda^{k}$

The accuracy of this approximation depends mainly on the strength of the gaseous absorption, aerosol loading, surface albedo, solar zenith angle, and observation geometry. As an example, Fig. 1 shows the term $\delta t_{p}(\lambda)$ and its approximation by a polynomial of second order obtained for space borne observations of the backscattered solar radiation in nadir viewing geometry at solar zenith angles of $60^{\circ}$ and $20^{\circ}$ in 320 $340 \mathrm{~nm}$ spectral range. Calculations were performed for the Rayleigh scattering coefficient and surface albedo increased by $10 \%$, and the aerosol extinction coefficient increased by 10,15 , and $25 \%$. As expected, $\delta t_{p}(\lambda)$ shows the same spectral structures as the ozone absorption coefficient and the approximation by a polynomial performs better at longer wavelengths where the ozone absorption is weaker.

Substituting $\delta t_{p}(\lambda)$ as given by Eq. (61) and $\delta k_{\lambda}(z)=$ $k_{\lambda}(z)-\bar{k}_{\lambda}(z)$, Eq. (60) is rewritten as follows:

$$
\begin{aligned}
\ln I_{\lambda}(k)= & \ln I_{\lambda}(\bar{k})-\int_{0}^{H} w_{\bar{k}}(\lambda, z) \bar{k}_{\lambda}(z) d z \\
& +\int_{0}^{H} w_{\bar{k}}(\lambda, z) k_{\lambda}(z) d z+\sum_{k=0}^{N} a_{k} \lambda^{k} .
\end{aligned}
$$

Let us consider now the difference between the first and the second terms in the right hand side of this equation. To explain the meaning of this difference we come back to the Taylor series expansion of $\ln I_{\lambda}(k)$ as given by Eq. (58) and rewrite it setting $k_{\lambda}(z)=0$, i.e., $\delta k_{\lambda}(z)=k_{\lambda}(z)-\bar{k}_{\lambda}(z)=$ $-\bar{k}_{\lambda}(z)$. It follows that

$\ln I_{\lambda}(0)=\ln I_{\lambda}(\bar{k})-\int_{0}^{H} w_{\bar{k}}(\lambda, z) \bar{k}_{\lambda}(z) d z+\varepsilon_{\operatorname{lin}}(\lambda)$,

where $I_{\lambda}(0)$ is the intensity obtained for a non-absorbing (i.e, without gaseous absorption) atmosphere. Introducing $\ln \tilde{I}_{\lambda}(0)$ as

$\ln \tilde{I}_{\lambda}(0)=\ln I_{\lambda}(\bar{k})-\int_{0}^{H} w_{\bar{k}}(\lambda, z) \bar{k}_{\lambda}(z) d z$

we obtain

$\ln I_{\lambda}(0)=\ln \tilde{I}_{\lambda}(0)+\varepsilon_{\operatorname{lin}}(\lambda)$.

As follows from this equation, $\ln \tilde{I}_{\lambda}(0)$ given by Eq. (64) is a linear estimation of the intensity logarithm for a nonabsorbing atmosphere. Combining Eqs. (62) and (64) we obtain

$\ln I_{\lambda}(k)=\int_{0}^{H} w_{\bar{k}}(\lambda, z) \sigma_{\lambda}(z) n(z) d z+\ln \tilde{I}_{\lambda}(0)+\sum_{k=0}^{N} a_{k} \lambda^{k}$,

where the product $\sigma_{\lambda}(z) n(z)$ is introduced instead of the absorption coefficient $k_{\lambda}(z)$. We note that in a general case the term $\ln \tilde{I}_{\lambda}(0)$ can not be approximated by a polynomial and, thus, must be kept in the right hand side of Eq. (66). This term can only be omitted if the linearization error $\varepsilon_{\operatorname{lin}}(\lambda)$ in Eq. (65) is negligible.

Equation (66) provides a linear relationship between the intensity logarithm and a gaseous absorber amount in a form of the absorber number density, $n(z)$, where a contribution of the scattering and reflection processes is approximated by a polynomial. Thus, according to the definition provided at the end of Sect. 2, this is a DOAS equation. In the following discussion Eq. (66) is referred to as the generalized DOAS equation. Introducing the slant column as

$S_{\lambda}=-\int_{0}^{H} w_{\bar{k}}(\lambda, z) n(z) d z$,

the gaseous absorber amount in Eq. (66) can be also represented by the slant column instead of the trace gas number density:

$\ln I_{\lambda}(k)=-S_{\lambda} \bar{\sigma}_{\lambda}+\ln \tilde{I}_{\lambda}(0)+\sum_{k=0}^{N} a_{k} \lambda^{k}$.

Here, $\bar{\sigma}_{\lambda}=\sigma_{\lambda}$ for a constant and

$$
\bar{\sigma}_{\lambda}=\frac{\int_{0}^{H} w_{\bar{k}}(\lambda, z) \sigma_{\lambda}(z) n(z) d z}{\int_{0}^{H} w_{\bar{k}}(\lambda, z) n(z) d z}
$$


for a spatially variable absorption cross section. In the following section we demonstrate that the slant column $S_{\lambda}$ introduced according to Eq. (67) is in line with its standard definition given by Eq. (12).

Concluding, let us summarize the assumptions which need to be employed to derive the generalized DOAS equation (the assumptions are required to hold in the considered spectral interval only):

- the gaseous absorption cross section has to contain a component rapidly varying with the wavelength;

- the wavelength dependence of non-gaseous (Rayleigh scattering, aerosol extinction, and surface albedo) weighting functions can be approximated by a polynomial of a low order;

- a local linearity of the intensity logarithm with respect to a variation of the gaseous absorber number density, $\delta n(z)$;

- a local linearity of the intensity logarithm with respect to variations of scattering and reflection parameters (Rayleigh scattering, aerosol extinction, and surface albedo).

\subsection{General expressions for the air mass factor and slant column}

In this section we introduce the most general expression for the air mass factor and demonstrate that the latter can be expressed via the weighting function, i.e., the variational derivative of the intensity logarithm with respect to the gaseous absorption coefficient introduced above. To this end let us start from Eq. (30) defining the box-AMF as a ratio of the partial slant column to the partial vertical column. Here, both slant and vertical columns are defined for finite light and vertical paths, $\left[l_{i-1}, l_{i}\right]$ and $\left[z_{i-1}, z_{i}\right]$, respectively. The air mass factor definition is generalized rewriting Eq. (30) for infinitesimally small light and vertical paths, denoted by $d l$ and $d z$, respectively. It follows that

$\mathcal{A}(\lambda, z)=\frac{d l(\lambda)}{d z}$,

where we have implicitly indicated that the light path $d l$ is a function of the wavelength. As clearly seen, $\mathcal{A}(\lambda, z)$ is a measure of the light path enhancement with respect to an infinitesimal vertical path $d z$ at an altitude $z$ due to the observation geometry and scattering processes. The main advantage of this definition with respect to that given by Eq. (30) is that it does not contain the absorber number density, $n(z)$.

To relate the air mass factor given by Eq. (70) to the weighting function (variational derivative) let us assume the absorption coefficient $\bar{k}_{\lambda}(z)$ to increase by $\delta k_{\lambda}(z)$ within an infinitesimal layer $d z$. Then, according to the Beer-Lambert law, the intensity logarithm for the absorption coefficient $k_{\lambda}(z)=\bar{k}_{\lambda}(z)+\delta k_{\lambda}(z)$ can be expressed as follows:

$\ln I_{\lambda}(k)=\ln I_{\lambda}(\bar{k})-\delta k_{\lambda}(z) d l(\lambda)$.

On the one hand, this equation can be rewritten in the following equivalent form:

$$
\begin{aligned}
\ln I_{\lambda}(k) & =\ln I_{\lambda}(\bar{k})-\delta k_{\lambda}(z) \frac{d l(\lambda)}{d z} d z \\
& =\ln I_{\lambda}(\bar{k})-\mathcal{A}(\lambda, z) \delta k_{\lambda}(z) d z,
\end{aligned}
$$

where $\mathcal{A}(\lambda, z)$ according to Eq. (70) is substituted. On the other hand, coming back to the functional Taylor series expansion of the intensity logarithm as given by Eq. (58) and recalling that the variation of the absorption coefficient $\delta k_{\lambda}(z)$ is assumed to be non-zero only within an infinitesimal layer $d z$, we rewrite this equation as follows:

$\ln I_{\lambda}(k)=\ln I_{\lambda}(\bar{k})+w_{\bar{k}}(\lambda, z) \delta k_{\lambda}(z) d z$.

The linearization error is neglected here. Comparing this equation to Eq. (72), it is obvious that

$\mathcal{A}(\lambda, z)=-w_{k}(\lambda, z)=-\left.\frac{\delta \ln I_{\lambda}}{\delta k_{\lambda}(z)}\right|_{\bar{k}_{\lambda}(z)}$.

Thus, in general, the air mass factor for an infinitesimal layer $d z$ is expressed via the variational derivative of the intensity logarithm with respect to the gaseous absorption coefficient. Other equivalent representations for the air mass factor are listed in Table 2. Assuming the variation of the gaseous absorption coefficient to be caused only by a variation of the trace gas number density, i.e., $\delta \sigma_{\lambda}=0$, the second representation is obvious. Derivations of the third and the fourth expressions containing the variational derivatives of the intensity logarithm with respect to the gaseous absorber optical depth and vertical column, respectively, are provided in Appendix B.

Let us demonstrate now that the slant column $S_{\lambda}$ introduced in the previous section by Eq. (67) is equivalent to its standard definition provided by Eq. (12). This is done substituting into Eq. (67) first $w_{\bar{k}}(\lambda, z)$ according to Eq. (74) and then $\mathcal{A}(\lambda, z)$ according to Eq. (70):

$$
\begin{aligned}
S_{\lambda} & =-\int_{0}^{H} w_{\bar{k}}(\lambda, z) n(z) d z=\int_{0}^{H} \mathcal{A}(\lambda, z) n(z) d z \\
& =\int_{0}^{H} \frac{d l(\lambda)}{d z} n(z) d z=\int_{l_{1}}^{l_{2}} n(l) d l(\lambda) .
\end{aligned}
$$

This means that the slant columns defined by Eqs. (12) and (67) are equivalent. A major advantage of the latter definition is, however, that the slant column can be calculated integrating the product of the weighting function and the absorber number density along the vertical direction over the entire atmosphere, i.e., without an explicit knowledge of photon paths. 
Table 2. General expressions for the air mass factor.

\begin{tabular}{lll}
\hline Num & Analytical form of $\mathcal{A}(\lambda, z)$ & Variational derivative with respect to \\
\hline 1 & $-\left.\frac{\delta \ln I_{\lambda}}{\delta k_{\lambda}(z)}\right|_{\bar{k}_{\lambda}(z)}$ & absorption coefficient \\
2 & $-\left.\frac{1}{\sigma \lambda} \frac{\delta \ln I_{\lambda}}{\delta n(z)}\right|_{\bar{n}(z)}$ & absorber number density \\
3 & $-\left.\int_{0}^{z} \frac{\delta \ln I_{\lambda}}{\delta \tau\left(z^{\prime}\right)}\right|_{\bar{\tau}\left(z^{\prime}\right)} d z^{\prime}$ & absorber optical depth \\
4 & $-\left.\frac{1}{\sigma \lambda} \int_{0}^{z} \frac{\delta \ln I_{\lambda}}{\delta V\left(z^{\prime}\right)}\right|_{\bar{V}\left(z^{\prime}\right)} d z^{\prime}$ & absorber vertical column \\
\hline
\end{tabular}

\section{Weighting function DOAS equation}

From the mathematical point of view the generalized DOAS equation given by Eq. (66) is a Fredholm integral equation of the first kind (Zabreiko et al., 1958; Widom, 1969). It contains the vertical profile of the absorbing gas number density, $n(z)$, as unknown parameter and is similar to Eq. (9) derived for observations of the direct solar light transmitted through the atmosphere. An approximative solution of such kind of equations can be found parameterizing the unknown variable, i.e., replacing a continuous function by a set of scalar parameters. Here, we consider a vertically inhomogeneous medium consisting of $N_{L}$ altitude layers within each of that the absorber number density is represented by the partial scaling approximation:

$n(z)=C_{i} \bar{n}(z), \quad z \in\left[z_{i-1}, z_{i}\right]$,

where $C_{i}$ is the number density scaling factor associated with the altitude layer $\left[z_{i-1}, z_{i}\right]$. Taking into account Eq. (14) the partial scaling approximation given by Eq. (76) can be rewritten as follows:

$n(z)=V_{i} \frac{\bar{n}(z)}{\bar{V}_{i}}, \quad z \in\left[z_{i-1}, z_{i}\right]$,

where $\bar{V}_{i}$ is the partial vertical column calculated for a priori number density profile $\bar{n}(z)$. Let us now rewrite the generalized DOAS equation given by Eq. (66) in the following equivalent form:

$$
\begin{aligned}
\ln I_{\lambda, j}(k)= & \sum_{i=1}^{N_{L}} \int_{z_{i-1}}^{z_{i}} w_{\bar{k}, j}(\lambda, z) \sigma_{\lambda}(z) n(z) d z \\
& +\ln \tilde{I}_{\lambda, j}(0)+\sum_{k=0}^{N} a_{k} \lambda^{k}
\end{aligned}
$$

where the subscript " $j$ " is restored to emphasize the dependence on observation conditions. The altitudes $z_{0}=0$ and $z_{N_{L}}=H$ denote the bottom and the top of the atmosphere, respectively, and the summation is performed over all altitude layers. Substituting $n(z)$ as given by Eq. (77) into Eq. (78) and introducing the layer-integrated weighting function as

$W_{i, j}(\lambda)=\frac{1}{\bar{V}_{i}} \int_{z_{i-1}}^{z_{i}} w_{\bar{k}, j}(\lambda, z) \sigma_{\lambda}(z) \bar{n}(z) d z$

we obtain:

$\ln I_{\lambda, j}(k)=\sum_{i=1}^{N_{L}} V_{i} W_{i, j}(\lambda)+\ln \tilde{I}_{\lambda, j}(0)+\sum_{k=0}^{N} a_{k} \lambda^{k}$.

This equation will be further referred to as the weighting function DOAS (WFDOAS) equation.

Multiplying and dividing the first term in the right hand side of Eq. (80) by the vertical path weighted cross section, $\hat{\sigma}_{\lambda}$, defined by Eq. (24), the WFDOAS equation can be rewritten in a form similar to the standard DOAS equation given by Eq. (34):

$\ln I_{\lambda, j}(k)=\left[\sum_{i=1}^{N_{L}} V_{i} \frac{W_{i, j}(\lambda)}{\hat{\sigma}_{\lambda}}\right] \hat{\sigma}_{\lambda}+\ln \tilde{I}_{\lambda, j}(0)+\sum_{k=0}^{N} a_{k} \lambda^{k}$.

Looking at Eqs. (81) and (34) one sees that the slant column appropriate to the WFDOAS equation can be expressed as

$S_{j}(\lambda)=-\sum_{i=1}^{N_{L}} V_{i} \frac{W_{i, j}(\lambda)}{\hat{\sigma}_{\lambda}}$.

Comparing further Eqs. (82) and (37) the corresponding relation for the box-AMF follows:

$A_{i, j}(\lambda)=-\frac{1}{\hat{\sigma}_{\lambda}} W_{i, j}(\lambda)$.

Using the definition of the layer-integrated weighting function given by Eq. (79) the box-AFM corresponding to the WFDOAS equation, as introduced by Eq. (83), can be also expressed as

$A_{i, j}(\lambda)=-\frac{1}{\hat{\sigma}_{\lambda} \bar{V}} \int_{z_{i-1}}^{z_{i}} w_{\bar{k}, j}(\lambda, z) \sigma_{\lambda}(z) \bar{n}(z) d z$. 
Employing Eq. (74), the absorption coefficient weighting function, $w_{\bar{k}, j}(\lambda, z)$, can be replaced by the AMF for an infinitesimal layer $d z$ :

$$
A_{i, j}(\lambda)=\frac{1}{\hat{\sigma}_{\lambda} \bar{V}_{i}} \int_{z_{i-1}}^{z_{i}} \mathcal{A}_{j}(\lambda, z) \sigma_{\lambda}(z) \bar{n}(z) d z,
$$

i.e., the box-AMF is represented as the layer-integrated AMF for an infinitesimal layer.

Substituting Eq. (83) into Eq. (81) the WFDOAS equation is rewritten as

$$
\ln I_{\lambda, j}(k)=-\left[\sum_{i=1}^{N_{L}} V_{i} A_{i, j}(\lambda)\right] \hat{\sigma}_{\lambda}+\ln \tilde{I}_{\lambda, j}(0)+\sum_{k=0}^{N} a_{k} \lambda^{k} .
$$

As can be seen here, contrastingly to the standard DOAS equation, the WFDOAS equation contains an additional term, $\ln \tilde{I}_{\lambda, j}(0)$, describing the linear estimation of the intensity logarithm for a non-absorbing atmosphere, and the appropriate box-AMF as given by Eq. (85) does not coincide with the commonly used expression given by Eq. (32).

We note that the usage of the DOAS technique in combination with weighting functions (WFDOAS) has been originally suggested by Buchwitz et al. (2000) to derive vertical columns of such gaseous absorber as $\mathrm{CO}, \mathrm{CO}_{2}, \mathrm{CH}_{4}$, and $\mathrm{N}_{2} \mathrm{O}$ from measurements of the backscattered solar radiation in the near infrared spectral range. Further, the WFDOAS approach has been successfully applied by Coldewey-Egbers et al. (2005) and Weber (2005) to retrieve vertical columns of ozone from GOME observations. An application of the WFDOAS technique to the retrieval of vertical profiles of $\mathrm{NO}_{2}$ and $\mathrm{BrO}$ from measurements of the scattered solar radiation in limb viewing geometry has been presented by Rozanov et al. (2005a).

Concluding, let us summarize the assumptions which need to be employed to derive the WFDOAS equation (the assumptions are required to hold in the considered spectral interval only):

- the gaseous absorption cross section has to contain a component rapidly varying with the wavelength;

- the wavelength dependence of non-gaseous (Rayleigh scattering, aerosol extinction, and surface albedo) weighting functions can be approximated by a polynomial of a low order;

- the partial scaling approximation for the vertical profile of the absorbing species;

- a local linearity of the intensity logarithm with respect to a variation of the partial vertical column, $\Delta V_{i}$;

- a local linearity of the intensity logarithm with respect to variations of scattering and reflection parameters (Rayleigh scattering, aerosol extinction, and surface albedo).
It is worth noticing here that besides the representation given by Eq. (85) the box-AMF corresponding to the WFDOAS equation can be also expressed via the partial derivative of the intensity logarithm with respect to the partial vertical column $\left(\partial \ln I / \partial V_{i}\right)$ or with respect to the layer optical thickness $\left(\partial \ln I / \partial \tau_{i}\right)$ of the absorbing species. Several representations for the box-AMF appropriate to the WFDOAS equation as given by Eq. (86) are listed in Table 3. As discussed above analytical expressions for $\mathcal{A}_{j}(\lambda, z)$ can be found in Table 2. For numerical calculations a finite-difference representation for the partial derivatives in the second and third expressions can be used. Further details thereon and derivation of these equations can be found in Appendix C.

\section{Modified DOAS equation}

As pointed out in Sect. 3 the modified DOAS (MDOAS) equation has been originally proposed by Diebel et al. (1994) and Fish et al. (1995) to improve the quality of trace gas vertical columns retrieved using the DOAS technique. In these studies the MDOAS equation has been obtained substituting a wavelength dependent slant column into the standard DOAS equation. Advantages of the former with respect to the standard DOAS equation for the interpretation of satellite measurements of the backscattered solar radiation in the ultraviolet and visible spectral ranges have been demonstrated among others by Diebel et al. (1994) performing synthetic retrievals of $\mathrm{O}_{3}$ and $\mathrm{NO}_{2}$ vertical columns.

In this section we derive the MDOAS equation starting from the WFDOAS equation. The latter has been obtained in Sect. 6 assuming the linearization error to be negligible in the case of a perturbed partial vertical column, $V_{i}=\bar{V}_{i}+\Delta V_{i}$, being within a small range around its a priori value, $\bar{V}_{i}$. For further consideration we exploit the relation between the layer integrated weighting function and the partial derivative of the intensity logarithm with respect to the partial vertical column written in the finite-difference representation as derived in Appendix C, see Eq. (C5). According to this expression the layer integrated weighting function is obtained from the difference in the intensity logarithm at two values of the partial column, namely, $V_{1}=\bar{V}_{i}$ and $V_{2}=\bar{V}_{i}+\Delta V_{i}$. Let us now calculate the partial derivative using the intensity logarithms at $V_{1}=0$, i.e., in a non-absorbing atmosphere, and at $V_{2}=\bar{V}_{i}$, i.e., including a priori partial vertical column of the absorber in the $i$-th altitude layer. Doing this Eq. (C5) for the layer-integrated weighting function results in

$W_{i, j}^{m}(\lambda)=-\frac{\ln I_{\lambda, j}(0)-\ln I_{\lambda, j}\left(\bar{V}_{i}\right)}{\bar{V}_{i}}$.

In general, weighting functions calculated according to Eqs. (C5) and (87) are different. To emphasize this fact we introduce the superscript " $m$ " for the weighting function calculated according to Eq. (87). 
Table 3. Expressions for the box-AMF appropriate to the WFDOAS equation, Eq. (86).

\begin{tabular}{cccc}
\hline Num & \multicolumn{4}{c}{ Analytical form of the box-AMF, $A_{i, j}(\lambda)$} & \\
& spatially variable cross section & constant cross section & given by \\
\hline 1 & $\frac{1}{\bar{V}_{i} \hat{\sigma}_{\lambda}} \int_{z_{i-1}}^{z_{i}} \mathcal{A}_{j}(\lambda, z) \sigma_{\lambda}(z) \bar{n}(z) d z$ & $\frac{1}{\bar{V}_{i}} \int_{z_{i-1}}^{z_{i}} \mathcal{A}_{j}(\lambda, z) \bar{n}(z) d z$ & Eq. (85) \\
2 & $-\left.\frac{1}{\hat{\sigma}_{\lambda}} \frac{\partial \ln I_{\lambda, j}}{\partial V_{i}}\right|_{\bar{V}_{i}}$ & $-\left.\frac{1}{\sigma_{\lambda}} \frac{\partial \ln I_{\lambda, j}}{\partial V_{i}}\right|_{\bar{V}_{i}}$ & Eq. (C6) \\
3 & $-\left.\frac{1}{\hat{\sigma}_{\lambda}} \frac{\partial \ln I_{\lambda, j}}{\partial \tau_{i}}\right|_{\bar{\tau}_{i}} \frac{\bar{\tau}_{i}}{\bar{V}_{i}}$ & $-\left.\frac{\partial \ln I_{\lambda, j}}{\partial \tau_{i}}\right|_{\bar{\tau}_{i}}$ & Eq. (C10) \\
\hline
\end{tabular}

Let us rewrite now the WFDOAS equation given by Eq. (80) to contain the layer-integrated weighting function $W_{i, j}^{m}(\lambda)$ as defined by Eq. (87):

$$
\begin{aligned}
\ln I_{\lambda, j}(k)= & \sum_{i=1}^{N_{L}} V_{i}\left[W_{i, j}^{m}(\lambda)+\Delta W_{i, j}(\lambda)\right]+\ln \tilde{I}_{\lambda, j}(0) \\
& +\sum_{k=0}^{N} a_{k} \lambda^{k}-\varepsilon_{\operatorname{lin}}(\lambda,-\bar{V}) .
\end{aligned}
$$

Here, $\Delta W_{i, j}(\lambda)=W_{i, j}(\lambda)-W_{i, j}^{m}(\lambda)$ is the difference between the weighting functions calculated according to Eqs. (C5) and (87). For further consideration we restore the linearization error, $\varepsilon_{\text {lin }}$, and indicate explicitly its dependence on the variation of the absorber vertical column (i.e., on $V-\bar{V}$ ). Taking into account that, according to Eq. (65),

$\ln \tilde{I}_{\lambda, j}(0)=\ln I_{\lambda, j}(0)-\varepsilon_{\operatorname{lin}}(\lambda,-\bar{V})$

and $\ln I_{\lambda, j}(0)$ can be approximated by a polynomial, Eq. (88) results in

$\ln I_{\lambda, j}(k)=\sum_{i=1}^{N_{L}} V_{i} W_{i, j}^{m}(\lambda)+\sum_{k=0}^{N} a_{k} \lambda^{k}+\varepsilon_{\operatorname{lin}}^{m}(\lambda, V-\bar{V})$,

where we introduce the linearization error of the MDOAS equation as

$$
\begin{aligned}
\varepsilon_{\operatorname{lin}}^{m}(\lambda, V-\bar{V}) \equiv & \sum_{i=1}^{N_{L}} V_{i} \Delta W_{i, j}(\lambda)-\varepsilon_{\operatorname{lin}}(\lambda,-\bar{V}) \\
& +\varepsilon_{\operatorname{lin}}(\lambda, V-\bar{V}) .
\end{aligned}
$$

As seen from Eq. (91), besides the linearization error of the WFDOAS equation, $\varepsilon_{\operatorname{lin}}(\lambda, V-\bar{V})$, the linearization error of the MDOAS equation, $\varepsilon_{\operatorname{lin}}^{m}(\lambda, V-\bar{V})$, contains two additional error terms. This fact explains different behavior of the errors discussed in Sect. 12.

Neglecting the linearization error Eq. (90) is rewritten as

$\ln I_{\lambda, j}(k)=\sum_{i=1}^{N_{L}} V_{i} W_{i, j}^{m}(\lambda)+\sum_{k=0}^{N} a_{k} \lambda^{k}$,

where $W_{i, j}^{m}(\lambda)$ is given by Eq. (87). Equation (92) will be further referred to as the modified DOAS (MDOAS) equation.
Multiplying and dividing the first term in the right hand side of the MDOAS equation by the vertical path weighted cross section, $\hat{\sigma}_{\lambda}$, defined by Eq. (24), Eq. (92) is rewritten as

$\ln I_{\lambda, j}(k)=-\sum_{i=1}^{N_{L}} V_{i} A_{i, j}(\lambda) \hat{\sigma}_{\lambda}+\sum_{k=0}^{N} a_{k} \lambda^{k}$,

where the appropriate box-AMF is defined by

$A_{i, j}(\lambda)=-\frac{1}{\hat{\sigma}_{\lambda}} W_{i, j}^{m}(\lambda)=\frac{\ln I_{\lambda, j}(0)-\ln I_{\lambda, j}\left(\bar{V}_{i}\right)}{\hat{\sigma}_{\lambda} \bar{V}_{i}}$.

Equation (93) is equivalent to Eq. (38) introduced in Sect. 3 whereas Eq. (94) defining the box-AMF appropriate to the MDOAS equation coincides with a commonly used definition of the box-AMF given by Eq. (32). Introducing the corresponding slant column as

$S_{j}(\lambda)=-\frac{1}{\hat{\sigma}_{\lambda}} \sum_{i=1}^{N_{L}} V_{i} W_{i, j}^{m}(\lambda)$

the MDOAS equation can also be rewritten in the following form:

$\ln I_{\lambda, j}(k)=-S_{j}(\lambda) \hat{\sigma}_{\lambda}+\sum_{k=0}^{N} a_{k} \lambda^{k}$,

where the gaseous absorber amount is characterized by its slant column.

Concluding, we state that the same assumptions as formulated in the previous section for the WFDOAS equation need to be employed to derive the modified DOAS equation. However, unlike the WFDOAS, the MDOAS equation does not contain $\ln \tilde{I}_{\lambda, j}(0)$ term and the appropriate box-AMF is obtained in a different way.

\section{Wavelength independent AMF for the standard DOAS equation}

As pointed out in Sect. 3, the standard DOAS technique requires a wavelength independent $\mathrm{AMF}$ to be defined whereas most of the previous studies report wavelength dependent AMFs suggesting, however, no unambiguous mapping rule to convert these to single values. In this section we derive 
a specific equation to calculate a wavelength independent AMF corresponding to the standard DOAS equation. For a simplicity reason we restrict our consideration to the total air mass factor, see Eq. (31) for the relation between the total and box AMFs. For the total column of an absorbing species the modified DOAS equation as given by Eq. (93) is rewritten as follows:

$\ln I_{\lambda, j}(k)=-V A_{j}(\lambda) \hat{\sigma}_{\lambda}+\sum_{k=0}^{N} a_{k} \lambda^{k}$.

Replacing in this equation the wavelength dependent air mass factor, $A_{j}(\lambda)$, by a constant value $A_{j}$, which is currently unknown, we have:

$\ln I_{\lambda, j}(k)=-V A_{j} \hat{\sigma}_{\lambda}+\sum_{k=0}^{N} a_{k} \lambda^{k}$.

We note that the polynomial coefficients in Eqs. (97) and (98) are different. The wavelength independent air mass factor, $A_{j}$, can be determined requiring Eqs. (97) and (98) to result in the same vertical column, $V$. Subtracting Eq. (97) from Eq. (98) and dividing both sides of the resulting equation by the vertical column, $V$, the following equation is obtained:

$A_{j}(\lambda) \hat{\sigma}_{\lambda}=A_{j} \hat{\sigma}_{\lambda}+\sum_{k=0}^{N} a_{k} \lambda^{k}$

Now all unknown parameters, namely, $A_{j}$ and polynomial coefficients, can be obtained employing the least square fit procedure. It is easy to demonstrate that the resulting constant AMF can be represented as a linear combination of $A_{j}(\lambda)$ at all discrete wavelengths in the considered spectral window. This is in line with results obtained by Marquard et al. (2000).

A practically more convenient equation to obtain the wavelength independent AMF can be derived substituting $A_{j}(\lambda)$ as given by Eq. (94) into Eq. (99):

$\frac{\ln I_{\lambda}(0)-\ln I_{\lambda}(\bar{V})}{\bar{V}}=A_{j} \hat{\sigma}_{\lambda}+\sum_{k=0}^{N} a_{k} \lambda^{k}$.

Multiplying both sides of this equation by $\bar{V}$ and taking into account that in a non-absorbing atmosphere the intensity logarithm can be approximated by a polynomial, the following equation is obtained:

$\ln I_{\lambda}(\bar{V})=-\bar{V} A_{j} \hat{\sigma}_{\lambda}+\sum_{k=0}^{N} a_{k} \lambda^{k}$.

Comparing this equation to Eq. (98) one can state that the former represents the standard DOAS equation written for a known value of the total column, $\bar{V}$. This means that the intensity logarithm in the left hand side of Eq. (101) is modeled for a known value of the total column, $\bar{V}$, whereas the term in the left hand side of Eq. (98) is the logarithm of the measured intensity corresponding to an unknown vertical column $V$.

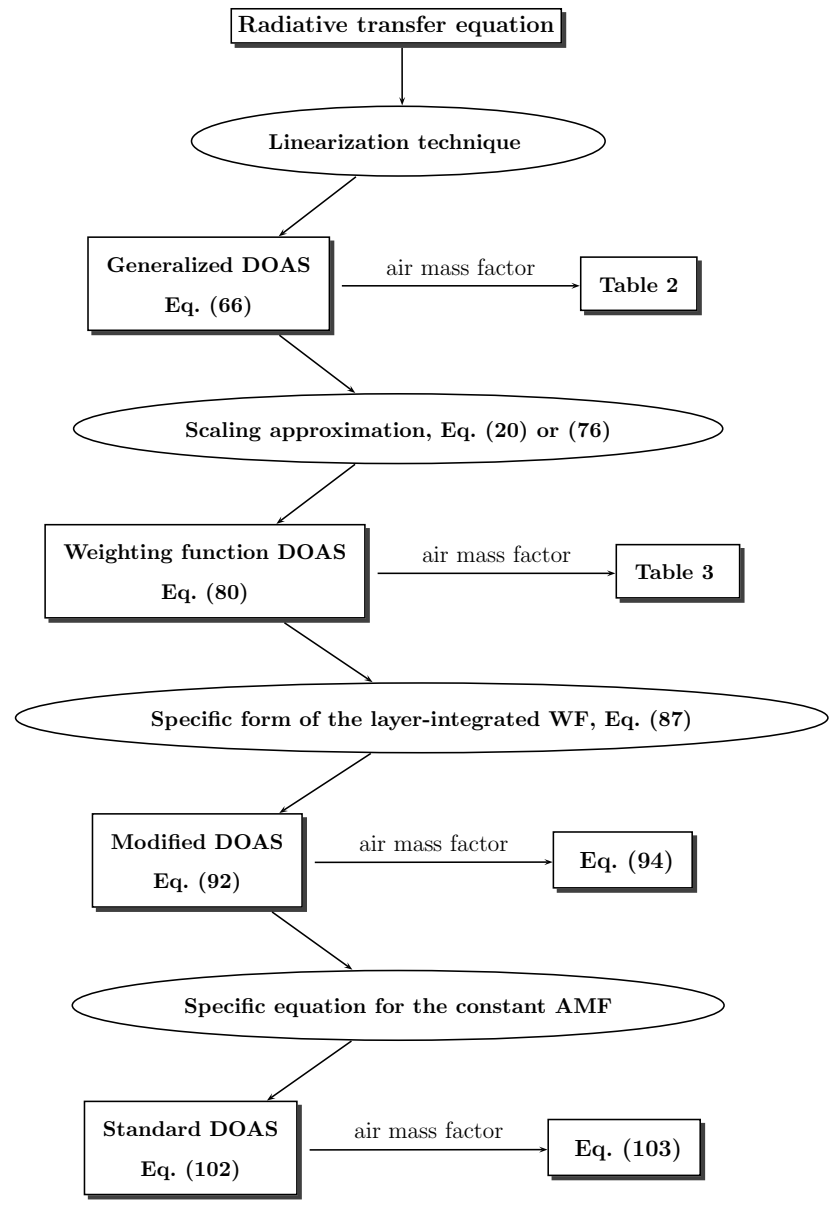

Fig. 2. Relationship between different DOAS equations.

Thus, the complete DOAS procedure to retrieve the vertical column is represented by the following system of equations:

$\ln I_{\lambda, j}(k)=-S_{j} \hat{\sigma}_{\lambda}+\sum_{k=0}^{N} a_{k} \lambda^{k}, j=1,2, \ldots, N_{S}$,

$A_{j}(\lambda) \hat{\sigma}_{\lambda}=A_{j} \hat{\sigma}_{\lambda}+\sum_{k=0}^{N} \beta_{k} \lambda^{k}$,

$S_{j}=V A_{j}$.

Here, $N_{s}$ is the number of observations performed at different conditions. As the system of Eqs. (102-104) was derived starting from the MDOAS equation, the appropriate expression for the wavelength dependent air mass factor, $A_{j}(\lambda)$, is given by Eq. (94).

Comparing these equations to the standard DOAS equation system given by Eqs. (34-37), it has been revealed that the recent formulation includes an explicit mapping rule to define the wavelength independent AMF, Eq. (103), instead 
Table 4. Assumptions that need to be employed to derive different DOAS equations suitable to interpret observations of the scattered solar light with a non-negligible contribution of the multiple scattering. The assumptions are required to hold in the considered spectral interval only.

\begin{tabular}{|c|c|c|c|c|c|}
\hline \multirow[t]{2}{*}{ Num } & \multirow[t]{2}{*}{ Assumptions } & \multicolumn{4}{|c|}{ DOAS equation } \\
\hline & & $\begin{array}{l}\text { generalized } \\
\text { Eq. (66) }\end{array}$ & $\begin{array}{l}\text { WF } \\
\text { Eq. }(80)\end{array}$ & $\begin{array}{l}\text { modified } \\
\text { Eq. }(92)\end{array}$ & $\begin{array}{l}\text { standard } \\
\text { Eq. (102) }\end{array}$ \\
\hline 1 & $\begin{array}{l}\text { the gaseous absorption cross section has to contain a component rapidly } \\
\text { varying with the wavelength }\end{array}$ & + & + & + & + \\
\hline 2 & $\begin{array}{l}\text { the wavelength dependence of weighting functions of scattering parameters } \\
\text { (Rayleigh scattering and aerosol extinction) and of the surface albedo can } \\
\text { be approximated by a polynomial of a low order }\end{array}$ & + & + & + & + \\
\hline 3 & $\begin{array}{l}\text { a local linearity of the intensity logarithm with respect to a variation of the } \\
\text { gaseous absorber number density, } \delta n(z)\end{array}$ & + & + & + & + \\
\hline 4 & $\begin{array}{l}\text { a local linearity of the intensity logarithm with respect to variations of scat- } \\
\text { tering and reflection parameters (Rayleigh scattering, aerosol extinction, } \\
\text { and surface albedo) }\end{array}$ & + & + & + & + \\
\hline 5 & $\begin{array}{l}\text { the partial scaling approximation for the vertical profile of the absorbing } \\
\text { species }\end{array}$ & $\begin{array}{c}\text { not } \\
\text { applied }\end{array}$ & + & + & + \\
\hline 6 & a specific form of the weighting function or air mass factor & \multicolumn{2}{|c|}{ not applied } & + & + \\
\hline 7 & $\begin{array}{l}\text { for a spatially variable absorption cross section the slant path weighted } \\
\text { cross section is assumed to be equal to the vertical path weighted cross } \\
\text { section }\end{array}$ & \multicolumn{3}{|c|}{ not applied } & + \\
\hline
\end{tabular}

of the symbolic link in the original formulation, Eq. (36). Clearly, as compared to the standard DOAS equation system, the usage of Eqs. (102-104) is computationally more expensive because one needs first to calculate the wavelength dependent $\mathrm{AMF}$ in the considered spectral window and then apply the least square fit to Eq. (103) to obtain the constant AMF. Therefore, in practical applications of the standard DOAS technique, different approximations are used to reduce the number of wavelengths at which the air mass factor needs to be calculated, see, e.g., Burrows et al. (1999); Marquard et al. (2000).

\section{Relationship between different DOAS equations}

In this section we discuss the relationship between the DOAS equations obtained previously. To facilitate the understanding, a flowchart shown in Fig. 2 illustrates the steps needed to derive different DOAS equations and Table 4 summarizes the assumptions which need to be employed when deriving these.

As seen from the flowchart in Fig. 2, the derivation of all DOAS equations begins with the formulation of the radiative transfer equation that provides a relationship between the intensity of the radiation and the atmospheric composition. A solution of the radiative transfer equation yields the intensity and its variational derivatives (weighting functions) with respect to atmospheric parameters for an assumed (a priori) state of the atmosphere. Obtained in this manner intensities and weighting functions contain already information on observation and illumination conditions, i.e., on the observation geometry, providing a basis to calculate AMF for any DOAS equation. As a discussion of the formulation and methods to solve the radiative transfer equation is outside the scope of this paper, we consider the intensity and weighting functions to be available. Having obtained these, the linearization technique based on the Taylor series expansion of the intensity logarithm is employed. Doing this we obtain a linear relationship between a variation of the intensity logarithm and variations of all relevant atmospheric parameters, see Eq. (59). If the requirements 1 and 2 listed in Table 4 are satisfied, this linear relationship provides the most general form of the DOAS equation referred to as the generalized DOAS equation, see Eq. (66). Although requirements 3 and 4 are not mandatory when deriving the generalized DOAS equation, a significant linearization error might occur if these requirements are not fulfilled.

The flowchart in Fig. 2 shows further that the WFDOAS equation is obtained from the generalized DOAS equation 
representing the vertical profile of the absorber number density by a discrete set of parameters rather than by a continuous function. In this study, the set of discrete parameters was obtained employing the partial scaling approximation as given by Eq. (76). Clearly, as the WFDOAS equation is derived from the generalized DOAS equation, it requires the same assumptions (1-4 as listed in Table 4) to be employed. Although a local linearity of the intensity logarithm with respect to a variation of the partial vertical column is needed to derive the WFDOAS equation, it does not need to be explicitly required because it follows from a more general assumption (see 3 in Table 4).

Employing a specific form of the partial derivative of the intensity logarithm with respect to the partial vertical column, see Eq. (87), the WFDOAS equation is transformed into the MDOAS equation, Eq. (92). The latter does not contain the term $\ln \tilde{I}_{\lambda, j}(0)$ any more which represents the linear estimation of the intensity logarithm for a non-absorbing atmosphere and is typical for the WFDOAS equation. It is worth noticing here, that the linearization errors of the WFDOAS and MDOAS equations are different, see Eq. (91), which leads to a different performance of these equations, see Sect. 12 for details. Clearly, the MDOAS equation requires the same initial assumptions as the WFDOAS equation, see Table 4.

Following further the flowchart shown in Fig. 2 one sees that the standard DOAS equation can be obtained rewriting the modified DOAS equation to contain the absorber slant column as the unknown parameter and requiring the latter to be independent of the wavelength. In this case, however, a wavelength independent air mass factor is needed to convert the resulting slant column into the vertical column. Thus, a specific rule to map the wavelength dependent air mass factor into an appropriate constant value needs to be established, see, e.g., Eq. (99).

\section{DOAS equations for a weakly absorbing media}

The standard DOAS technique is commonly used assuming weak gaseous absorption. However, related publications usually do not provide any quantitative criterion of the absorption weakness. In this section we establish such a criterion and demonstrate that all DOAS equations considered previously become nearly equivalent if this criterion is satisfied.

\subsection{Quantitative criterion of a weak absorption}

Obviously, the strength of the gaseous absorption is determined both by the amount of the absorbing gas in the atmosphere and by the light path. For observations of the scattered solar light with a non-negligible contribution of the multiple scattering the actual light path is unknown and is determined by the absorption, scattering and reflection properties of the Earth's atmosphere and surface. To take all these effects into account, the error of the linear estimation of the intensity logarithm for a non-absorbing atmosphere can be used as a measure of an absorption weakness. According to Eq. (65) this error is written as

$\varepsilon_{\operatorname{lin}, j}(\lambda)=\ln I_{\lambda, j}(0)-\ln \tilde{I}_{\lambda, j}(0)$,

where " $j$ " denotes observation/illumination conditions, $\ln I_{\lambda, j}(0)$ is the intensity logarithm for a non-absorbing atmosphere, and $\ln \tilde{I}_{\lambda, j}(0)$ is its linear estimation according to Eq. (64). Rewriting the latter as

$\ln \tilde{I}_{\lambda, j}(0)=\ln I_{\lambda, j}(\bar{k})-\int_{0}^{H} w_{\bar{k}, j}(\lambda, z) \sigma_{\lambda}(z) \bar{n}(z) d z$

and substituting it into Eq. (105) we obtain

$\varepsilon_{\operatorname{lin}, j}(\lambda)=\ln I_{\lambda, j}(0)-\left[\ln I_{\lambda, j}(\bar{k})-\int_{0}^{H} w_{\bar{k}, j}(\lambda, z) \sigma_{\lambda}(z) \bar{n}(z) d z\right]$.

Taking into account the definition of the layer-integrated weighting function given by Eq. (79), we rewrite Eq. (107) in the following equivalent form:

$\varepsilon_{\operatorname{lin}, j}(\lambda)=\ln I_{\lambda, j}(0)-\left[\ln I_{\lambda, j}(\bar{k})-\bar{V} W_{j}(\lambda)\right]$,

where the weighting function for the entire atmosphere $W_{j}(\lambda)$ is given by

$W_{j}(\lambda)=\frac{1}{\bar{V}} \int_{0}^{H} w_{\bar{k}, j}(\lambda, z) \sigma_{\lambda}(z) \bar{n}(z) d z$.

Thus, for a given a priori vertical column of the absorbing species, $\bar{V}$, and observation/illumination conditions, the gaseous absorption can be considered to be weak if $\varepsilon_{\operatorname{lin}, j}(\lambda)$ is smaller than a certain criterion, e.g., measurement error.

Figure 3 shows examples of the linearization error $\varepsilon_{\operatorname{lin}, j}(\lambda)$ (red curves) calculated according to Eq. (108) for spaceborne observations of the backscattered solar radiation performed in nadir viewing geometry at solar zenith angles of $70^{\circ}$ and $90^{\circ}$ in $320-330 \mathrm{~nm}$ and $425-455 \mathrm{~nm}$ spectral ranges containing absorption features of $\mathrm{O}_{3}$ and $\mathrm{NO}_{2}$, respectively. For a comparison, the differential slant optical thickness, $-L_{\lambda, j}^{d}(k)$, calculated according to Eq. (44) is shown with black curves in the same plots. The calculations were performed for the ozone vertical column of $343 \mathrm{DU}$ and $\mathrm{NO}_{2}$ vertical column of $3.47 \times 10^{15} \mathrm{~cm}^{-2}(0.13 \mathrm{DU})$. As expected, the linearization error depends not only on the absorber amount but also on the solar zenith angle. In particular, in the $425-455 \mathrm{~nm}$ spectral range the maximum absolute values of the linearization error reach about $\sim 3 \times 10^{-5}$ for both solar zenith angles and, thus, the gaseous absorption can be considered to be weak. In contrast, the linearization error in $320-330 \mathrm{~nm}$ spectral range can be significant even at a solar 

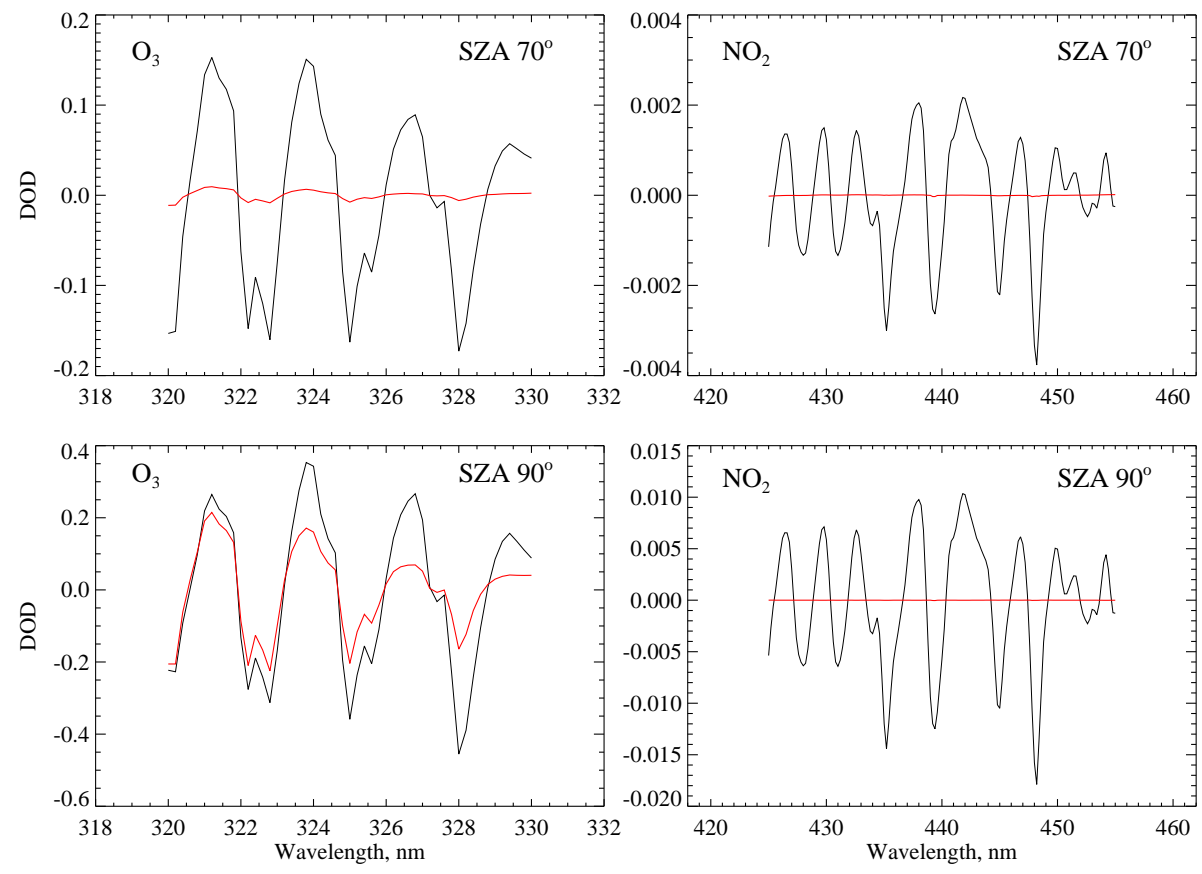

Fig. 3. Linearization error $\varepsilon_{\operatorname{lin}, j}(\lambda)$ according to Eq. (108) in absorption bands of $\mathrm{O}_{3}$ (left panels) and $\mathrm{NO}_{2}$ (right panels) at solar zenith angles of $70^{\circ}$ (upper panels) and $90^{\circ}$ (lower panels). Red curves show the linearization error whereas black curves denote the differential slant optical thickness of the absorbing species, $-L_{\lambda, j}^{d}(k)$, see Eq. (44).

zenith angle of $70^{\circ}$. At $90^{\circ}$ the magnitude of the linearization error becomes comparable with the differential slant optical thickness of ozone itself and, thus, the gaseous absorption is definitively not weak any more.

\subsection{Near-equivalence of different DOAS equations}

In this subsection we demonstrate that if the gaseous absorption can be considered to be weak, i.e., $\varepsilon_{\operatorname{lin}, j}(\lambda)$ given by Eq. (108) is smaller than a selected criterion, all previously considered DOAS equations are nearly equivalent. A prerequisite for a small linearization error is the near-linearity of the intensity logarithm as a function of the absorber number density in a wide range around its a priori value $\bar{n}(z)$. This assumption will be further referred to as a nearly global linearity. In contrast to the global linearity, it does not require the linear relationship between intensity logarithm and the absorber amount to be satisfied exactly. It is worth noticing here that in the case of the global linearity the Beer-Lambert law has to be complied exactly which is impossible for observations of the scattered light.

It is well known that the derivative of a linear function with respect to its argument is a constant. Hence, assuming a near-linearity, the derivative of the intensity logarithm with respect to the partial vertical column of the absorbing species is nearly constant and the finite-difference representation for the layer integrated weighting function, see Eq. (C5), is valid for almost any $\Delta V_{i}$. Setting $\Delta V_{i}=-\bar{V}_{i}$, Eq. (C5) is rewritten as
$W_{i, j}(\lambda)=-\frac{\ln I_{\lambda, j}(0)-\ln I_{\lambda, j}\left(\bar{V}_{i}\right)}{\bar{V}_{i}}$.

As clearly seen, the layer integrated weighting function resulting from this equation is exactly the same as that for the MDOAS equation, see Eq. (87). Furthermore, as the linearization error is small, the intensity logarithm for a nonabsorbing atmosphere, $\ln I_{\lambda, j}(0)$, is close to its linear estimation, $\ln \tilde{I}_{\lambda, j}(0)$, i.e., $\ln \tilde{I}_{\lambda, j}(0) \approx \ln I_{\lambda, j}(0)$, see Eq. (105). Thus, the latter can be approximated by a polynomial of a low order in Eq. (80). As clearly seen, the WFDOAS equation as given by Eq. (80) and the MDOAS equation as given by Eq. (92) become then identical.

As discussed in Appendix C, the air mass factor can be obtained as the derivative of the intensity logarithm with respect to the layer optical thickness of the absorber. Under assumption of the near-linearity this derivative depends only weakly on the absorber optical thickness. Thus, the resulting air mass factor is nearly independent of the wavelength and no additional efforts are needed to use it in the framework of the standard DOAS equation, i.e., the spectral fit according to Eq. (103) is unnecessary. Thus, the standard DOAS equation does not differ from the MDOAS equation any more.

Considering the generalized DOAS equation written for the slant column as a measure of the gaseous absorber amount, see Eq. (68), for a weakly absorbing medium we can state the following:

- as the weighting function depends on the wavelength and altitude only weakly, the absorption cross section 
Table 5. Alternative expressions for the air mass factor proposed in previous publications and appropriate DOAS equations.

\begin{tabular}{|c|c|c|c|}
\hline Num & Air mass factor & $\begin{array}{c}\text { DOAS } \\
\text { equation }\end{array}$ & Reference \\
\hline 1 & $\frac{\ln I_{\lambda}(0)-\ln I_{\lambda}(\bar{n})}{\sigma_{\lambda} \bar{V}}$ & $\begin{array}{l}\text { MDOAS } \\
\text { Eq. (92) }\end{array}$ & Perliski and Solomon (1993) Sarkissian et al. (1995) \\
\hline 2 & $-\frac{\partial \ln I_{\lambda}}{\partial \tau(\lambda)}$ & $\begin{array}{l}\text { WFDOAS } \\
\text { Eq. (80) }\end{array}$ & Slusser et al. (1996) \\
\hline 3 & $-\frac{1}{\sigma_{\lambda}} \frac{\partial \ln I_{\lambda}}{\partial V}$ & $\begin{array}{l}\text { WFDOAS } \\
\text { Eq. (80) }\end{array}$ & Stammes and Koelemeijer (1999) \\
\hline 4 & $-\frac{\mathcal{W}(\lambda)}{\bar{\tau}(\lambda) I_{\lambda}(\bar{k})}$ & $\begin{array}{l}\text { WFDOAS } \\
\text { Eq. (80) }\end{array}$ & Rozanov et al. (1998) \\
\hline 5 & $\frac{1}{\sigma_{\lambda}^{d} \bar{V}} \ln \frac{I_{\lambda}\left(\sigma_{\lambda}^{c}\right)}{I_{\lambda}\left(\sigma_{\lambda}\right)}$ & $\begin{array}{l}\text { WFDOAS } \\
\text { Eq. (80) }\end{array}$ & Marquard et al. (2000) \\
\hline 6 & $-\frac{1}{\bar{\tau}(\lambda)} \sum_{i=1}^{N_{L}}\left[\frac{\partial \ln I_{\lambda}}{\partial \tau_{i}(\lambda)} \sigma_{\lambda, i} \bar{n}_{i} \Delta z_{i}\right]$ & $\begin{array}{c}\text { WFDOAS } \\
\text { Eq. (80) }\end{array}$ & Palmer et al. (2001) \\
\hline
\end{tabular}

weighted according to Eq. (69) is close to the vertical path weighted cross section as given by Eq. (24);

- the slant column given by Eq. (67) is nearly independent of the wavelength;

- the term $\ln \tilde{I}_{\lambda}(0)$ in Eq. (68) describing the linear estimation of the intensity logarithm for a non-absorbing atmosphere can be approximated by a low order polynomial.

Thus, for a weakly absorbing medium the generalized DOAS equation is equivalent to the standard DOAS equation.

\section{Alternative expressions for the air mass factor}

In previous sections the WFDOAS and MDOAS equations as well as corresponding expressions for the air mass factor were derived. Here we consider alternative representations for the AMF proposed in previous publications and compare these to the expressions discussed above. For a simplicity reason we consider the total AMF only. Therefore, throughout this section the subscripts " $i$ " and " $j$ " denoting atmospheric layers and observation/illumination conditions, respectively, will be omitted.

As follows from the discussion above, the difference between various DOAS equations and corresponding AMFs is insignificant in a case of a weak gaseous absorption only. Otherwise, each DOAS equation has to be used along with the appropriate expression for the AMF. A usage of inappropriate air mass factor in a case of a non-weak absorption may lead to an increased retrieval error. Unfortunately, the authors of some previous publications considered here did not attribute their expressions for the AMF to any DOAS equations. For these expressions the correct attribution is derived below. All considered expressions for the air mass factor along with the dedicated DOAS equations are listed in Table 5 .

As the first expression for the AMF is discussed in Sect. 7 and the derivation of the second, third, and sixth, expressions is presented in Appendix $\mathrm{C}$, we consider here only the fourth and fifth expressions. The latter has been proposed by Marquard et al. (2000) in a form of

$A(\lambda)=\frac{1}{\sigma_{\lambda}^{d} \bar{V}} \ln \frac{I_{\lambda}\left(\sigma_{\lambda}^{c}\right)}{I_{\lambda}\left(\sigma_{\lambda}\right)}$,

where the absorption cross section, $\sigma_{\lambda}$, is supposed to be split into two terms, $\sigma_{\lambda}=\sigma_{\lambda}^{c}+\sigma_{\lambda}^{d}$, with $\sigma_{\lambda}^{c}$ smoothly dependent on the wavelength and $\sigma_{\lambda}^{d}$ comprising sharp spectral features. The term $\ln I_{\lambda}\left(\sigma_{\lambda}^{c}\right)$ denotes the intensity logarithm calculated assuming the absorption cross section to be $\sigma_{\lambda}^{c}$ instead of $\sigma_{\lambda}$. In order to find a relation between $A(\lambda)$ given by Eq. (111) and air mass factors discussed previously, let us expand $\ln I_{\lambda}\left(\sigma_{\lambda}\right)$ into the Taylor series with respect to a variation of the cross section, $\sigma_{\lambda}$, around $\sigma_{\lambda}^{c}$. As, in this case, a variation of the absorption coefficient is caused by a variation of the cross section only, i.e., $\delta k_{\lambda}(z)=k_{\lambda}(z)-\bar{k}_{\lambda}(z)=$ $\bar{n}(z)\left(\sigma_{\lambda}-\sigma_{\lambda}^{c}\right)$, the functional Taylor series for the intensity logarithm as given by Eq. (58) can be rewritten as follows: 
$\ln I_{\lambda}\left(\sigma_{\lambda}\right)=\ln I_{\lambda}\left(\sigma_{\lambda}^{c}\right)+\int_{0}^{H} w_{\bar{k}}(\lambda, z) \bar{n}(z) \delta \sigma_{\lambda} d z$.

Here, $\delta \sigma_{\lambda}=\sigma_{\lambda}-\sigma_{\lambda}^{c}$ and the linearization error, $\varepsilon_{\operatorname{lin}}(\lambda)$, is neglected. Taking into account that $\delta \sigma_{\lambda}=\sigma_{\lambda}^{d}$, we rewrite Eq. (112) in the following equivalent form:

$\ln \frac{I_{\lambda}\left(\sigma_{\lambda}\right)}{I_{\lambda}\left(\sigma_{\lambda}^{c}\right)}=\int_{0}^{H} w_{\bar{k}}(\lambda, z) \bar{n}(z) \sigma_{\lambda}^{d} d z$.

Substituting this expression into Eq. (111) and taking into account that the absorption cross section in Marquard et al. (2000) was assumed to be independent of the temperature and pressure (i.e., of the altitude), the following equation for the air mass factor is obtained:

$A(\lambda)=-\frac{1}{\bar{V}} \int_{0}^{H} w_{\bar{k}}(\lambda, z) \bar{n}(z) d z$.

On the other hand, integrating the layer-integrated weighting function, introduced by Eq. (79), over the entire atmosphere (i.e., setting $z_{i-1}=0$ and $z_{i}=H$ ) we have

$W(\lambda)=\frac{\sigma_{\lambda}}{\bar{V}} \int_{o}^{H} w_{\bar{k}}(\lambda, z) \bar{n}(z) d z$.

where, similar to Eq. (114), the cross section is considered to be independent of the altitude. Combining Eqs. (114) and Eq. (115) we obtain

$A(\lambda)=-\frac{W(\lambda)}{\sigma_{\lambda}}$.

As clearly seen, the air mass factor introduced in Marquard et al. (2000) as given by Eq. (111) is expressed in a linear approximation via the integrated weighting function and coincides with the AMF corresponding to the WFDOAS equation discussed above, see Eq. (83).

Another representation of the total AMF via the integrated weighting function has been suggested by Rozanov et al. (1998) in the following form (fourth entry in Table 5):

$A(\lambda)=-\frac{\mathcal{W}(\lambda)}{\bar{\tau}(\lambda) I_{\lambda}(\bar{k})}$.

Here, $\mathcal{W}(\lambda)$ is the variational derivative of the intensity with respect to the gaseous absorber number density integrated over the entire atmosphere and is given by

$\mathcal{W}(\lambda)=\left.\int_{0}^{H} \frac{\delta I_{\lambda}}{\delta n(z)}\right|_{\bar{n}(z)} \bar{n}(z) d z$,

$\bar{\tau}(\lambda)$ is the gaseous absorption optical thickness, and $I_{\lambda}(\bar{k})$ is the intensity calculated with a radiative transfer model for a priori number density profile, $\bar{n}(z)$, of the absorbing species. Recalling the definition of the absorption coefficient weighting function given by Eq. (57) and assuming the absorption coefficient to vary only due to a variation of the absorber number density, i.e., $\delta k_{\lambda}(z)=\sigma_{\lambda}(z) \delta n(z)$, we rewrite Eq. (118) as follows:

$\mathcal{W}(\lambda)=I_{\lambda}(\bar{n}) \int_{0}^{H} w_{\bar{k}}(\lambda, z) \sigma_{\lambda}(z) \bar{n}(z) d z$,

Extending the integration limits in Eq. (79) to the entire atmosphere and comparing it to Eq. (119) we obtain:

$W(\lambda)=\frac{\mathcal{W}(\lambda)}{\bar{V} I_{\lambda}(\bar{n})}$.

Thus, Eq. (117) results in

$A(\lambda)=-\frac{W(\lambda) \bar{V}}{\bar{\tau}(\lambda)}=-\frac{W(\lambda)}{\bar{\sigma}_{\lambda}}$

that coincides with the AMF corresponding to the WFDOAS equation discussed above, see Eq. (83).

\section{Performance of different DOAS equations}

In this section we investigate the performance of different DOAS equations employing the numerical experiment technique. The retrievals are performed for the total vertical column of ozone using simulated outgoing radiances at the top of the atmosphere in $320-330 \mathrm{~nm}$ spectral range. As discussed in Sect. 10, for this retrieval scenario the criterion of a weak absorption is not satisfied. Thus, different DOAS equations are expected to produce different results whereas in a case of a weak absorption all DOAS equations are nearly equivalent. The numerical experiments are performed as follows:

- the intensity of the backscattered solar radiation in $320-330 \mathrm{~nm}$ spectral range is simulated with the SCIATRAN radiative transfer model (Rozanov et al., 2005b; Rozanov, 2008) for various amounts of ozone in the atmosphere (30-700 DU total column) consistently with GOME (Burrows et al., 1999) observations in the nadir viewing geometry at solar zenith angles of $70^{\circ}$ and $90^{\circ}$;

- the simulated spectra are treated as synthetic experimental data obtained for a known vertical column of ozone which is further referred to as the true vertical column;

- the air mass factors (or weighting functions) are always calculated for a priori vertical column of ozone, $\bar{V}$ (other parameters are the same as for simulated measurements);

- the DOAS fit is performed for each synthetic observation employing different DOAS equations; 


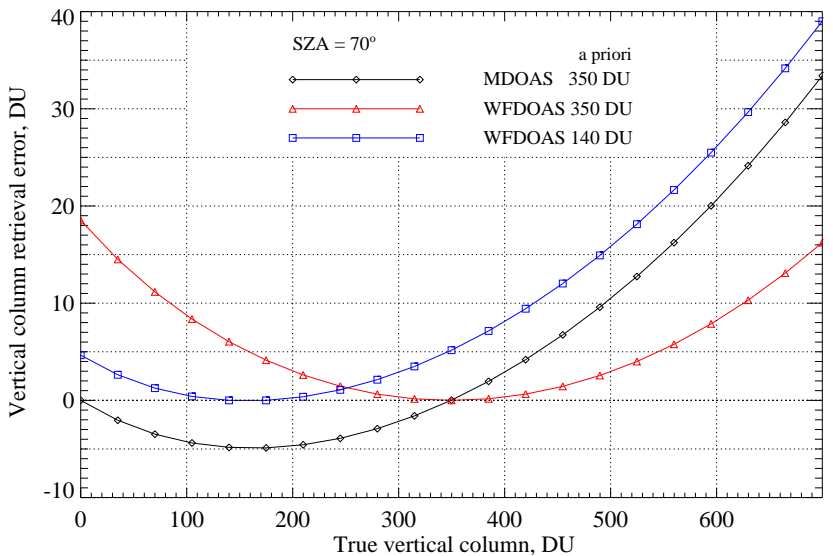

Fig. 4. Error in the retrieved vertical column of ozone as a function of the true vertical column for a solar zenith angle of $70^{\circ}$. The results of MDOAS and WFDOAS retrievals for a priori vertical column of $350 \mathrm{DU}$ are shown with black and red lines, respectively, whereas the errors resulting from WFDOAS equation for a priori vertical column of $140 \mathrm{DU}$ are shown with the blue line.

- absolute retrieval errors (defined as the difference between the true and the retrieved vertical columns) for different DOAS equations are analyzed.

It is worth noticing here that in the case under consideration the only source of error in the retrieval process is the linearization error.

\subsection{WFDOAS and MDOAS equations}

In this section errors in the ozone vertical columns retrieved employing the WFDOAS and MDOAS equations, as given by Eqs. (80) and (92), respectively, are compared. The retrieval errors for both equations as functions of the true vertical column of ozone are shown in Figs. 4 and 5 for solar zenith angles of $70^{\circ}$ and $90^{\circ}$, respectively. As clearly seen, for an a priori ozone vertical column of $350 \mathrm{DU}$, the WFDOAS approach (red line) is more accurate than the MDOAS (black line) for true vertical columns of ozone larger than about $170 \mathrm{DU}$ whereas the opposite behavior is observed for lower values of the true vertical column. As a decreased retrieval accuracy is associated to the linearization error, it can be significantly improved employing an iterative technique. The efficiency of this approach can be demonstrated using, for example, $140 \mathrm{DU}$, as a new a priori vertical column of ozone. Doing this the retrieval error associated with the WFDOAS approach decreases significantly for true vertical columns of ozone below about 230 DU (shown in Figs. 4 and 5 with blue lines).

The dependence of the retrieval error on the difference between the true and a priori vertical columns can be explained analyzing the linearization error of both DOAS equations. To this end let us rewrite WFDOAS and MDOAS equations as given by Eqs. (80) and (92), respectively, for the total vertical

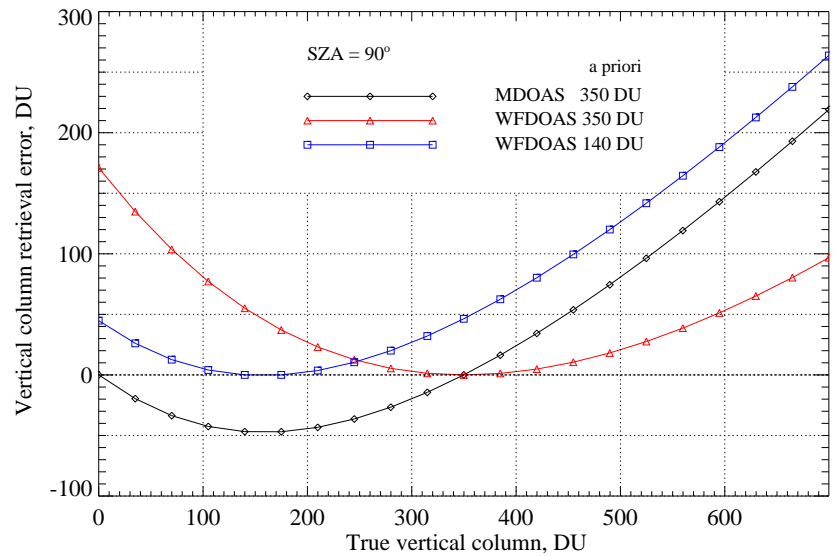

Fig. 5. Same as in Fig. 4 but for a solar zenith angle of $90^{\circ}$.

column of the absorbing species:

$\ln I_{\lambda}(V)=V W_{w}(\lambda, \bar{V})+\ln \tilde{I}_{\lambda}(0)+\sum_{k=0}^{N} a_{k} \lambda^{k}$,

$\ln I_{\lambda}(V)=V W_{m}(\lambda, \bar{V})+\sum_{k=0}^{N} a_{k} \lambda^{k}$.

Here, we omit the subscript " $j$ " denoting the observation/illumination conditions and introduce subscripts " $w$ " and " $m$ " designating the weighting functions attributed to WFDOAS and MDOAS equations, respectively. Additionally, we explicitly indicate the dependence of weighting functions on a priori vertical column, $\bar{V}$. The linearization error is defined as the difference between the left and the right hand sides of the corresponding equation. For the sake of simplicity we use the root mean squares of the linearization errors in the discussion below. Linearization errors associated to the WFDOAS and MDOAS equations are shown in Fig. 6 by the red and black lines, respectively. The errors are estimated at a solar zenith angle of $90^{\circ}$. As seen from the plot, the linearization error of the MDOAS equation touches the zero level twice, namely, at $V=0$ and $V=\bar{V}$. This fact is explained by the appearance of too additional error terms in the MDOAS equation. As seen from Eq. (91), error canceling occurs for $V=0$ leading to a better accuracy of the MDOAS equation at small values of the true vertical column. For true vertical columns larger than a priori value, however, the linearization error of the WFDOAS equation is always smaller than that of the MDOAS equation. A similar behavior but an order of magnitude smaller values are observed for the linearization errors at a solar zenith angle of $70^{\circ}$ (not shown here)

Thus, if no iterative technique is employed, the usage of the WFDOAS equation is preferable for true vertical columns arbitrary larger or less than 50\% lower than a priori vertical column whereas for true vertical columns much 


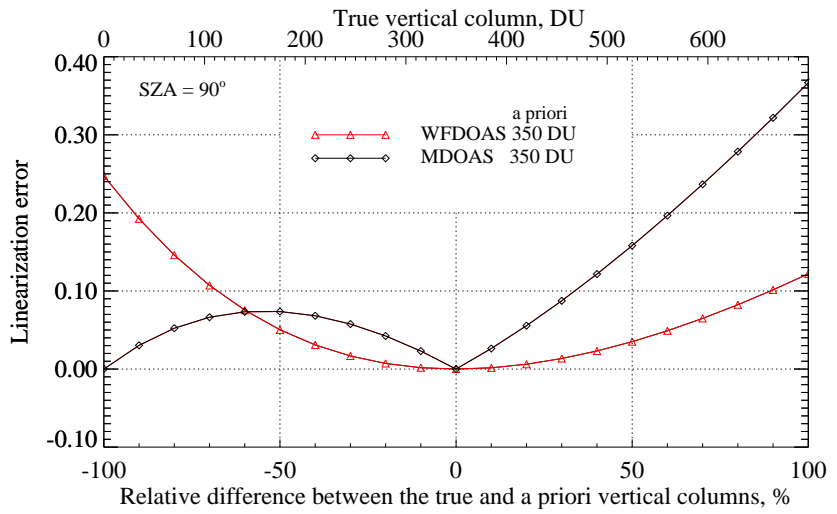

Fig. 6. Root mean square of the linearization error for WFDOAS (red line) and MDOAS (black line) equations at a solar zenith angle of $90^{\circ}$ for a priori ozone vertical column of 350 DU.

lower then a priori column the usage of the MDOAS equation can be advantageous.

\subsection{Standard DOAS equation}

The performance of the standard DOAS technique defined by Eqs. (102-104) is the same as for the MDOAS approach given by Eq. (97). This is due to the fact that the mapping of the wavelength dependent AMF associated to the MDOAS equation into a constant AMF needed for the standard DOAS equation is done requiring absorber vertical columns resulting from both equations to be the same. As discussed above, the error in the retrieved vertical columns is caused by the linearization error associated with the corresponding DOAS equation. Thus, similarly to the discussion above, the retrieval error associated to the standard DOAS technique can be substantially reduced using an iterative procedure which for the standard DOAS approach given by Eqs. (102-104) is written as follows:

$\ln I_{\lambda}(V)=-S \sigma_{\lambda}+\sum_{k=0}^{N} a_{k} \lambda^{k}$

$\ln I_{\lambda}\left(V_{n-1}\right)=-V_{n-1} A_{c}\left(V_{n}\right) \sigma_{\lambda}+\sum_{k=0}^{N} a_{k} \lambda^{k}$,

$V_{n}=\frac{S}{A_{c}\left(V_{n}\right)}$.

Here, $n$ is the iteration number starting with $n=1$, and $V_{0}=\bar{V}$ is a priori vertical column. The term $\ln I_{\lambda}(V)$ in Eq. (124) denotes the logarithm of the measured intensity whereas $\ln I_{\lambda}\left(V_{n-1}\right)$ in Eq. (125) is the logarithm of the modeled intensity calculated for the absorber vertical column of $V_{n-1}$. Hence, each iterative step comprises the calculation of $\ln I_{\lambda}\left(V_{n-1}\right)$ employing an appropriate radiative transfer model, DOAS fit according to Eq. (125) with respect to $A_{c}\left(V_{n}\right)$, and calculation of $V_{n}$ according to Eq. (126).

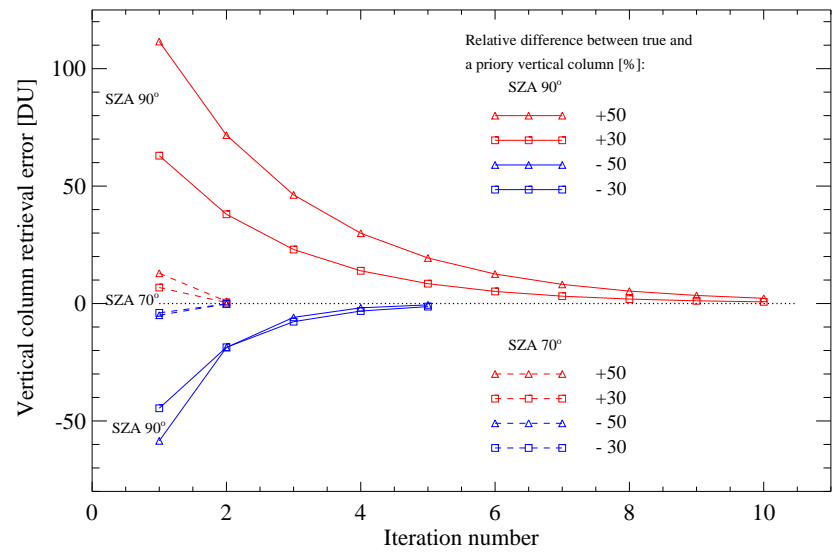

Fig. 7. Convergence rate of the iterative process according to Eqs. (124-126) for solar zenith angles of $70^{\circ}$ (dashed lines) and $90^{\circ}$ (solid lines). Symbols designate the retrieval errors obtained for selected percentage differences between the true and a priori vertical columns.

An important question arising when using an iterative approach is how quickly it converges. The convergence rate of the iterative process according to Eqs. (124-126) is illustrated in Fig. 7 for solar zenith angles of $70^{\circ}$ (dashed lines) and $90^{\circ}$ (solid lines). The calculations are performed for a priori ozone vertical column of $350 \mathrm{DU}$ and a set of true vertical columns. The retrieval errors are estimated for four selected percentage differences between the true, $V$, and a priori vertical columns, $\bar{V}$, defined as $\Delta(V)=(V-\bar{V}) / \bar{V}$. $100 \%$, namely, for $\Delta(V)= \pm 30 \%$ and $\Delta(V)= \pm 50 \%$. As clearly seen, for a solar zenith angle of $70^{\circ}$ the retrieval error is less than $\sim 1 \mathrm{DU}$ already at the second iterative step whereas for a solar zenith angle of $90^{\circ}$ the convergence rate is much lower. Here, 5 iterative steps for negative $\Delta(V)$ and 10 steps for positive $\Delta(V)$ are needed to reduce the retrieval error to $1 \mathrm{DU}$ level. This example clearly demonstrates that the retrieval error and the convergence rate depend not only on the magnitude of the difference between the true and a priori vertical columns but also on its sign. In particular, as seen from Fig. 7, the convergence rate is significantly lower for true vertical columns larger than a priori column. This behavior is a specific feature of the MDOAS equation which can be easily explained looking at the linearization error shown in Fig. 6. As clearly seen, the error is not symmetric with respect to a priori vertical column and is typically larger for positive differences between the true and a priori vertical columns.

Thus, similarly to the WFDOAS and MDOAS equations, the standard DOAS technique is capable to provide an accurate solution for the absorber vertical column. At large solar zenith angles, however, an iterative approach requiring multiple simulations of the radiance in the fitting spectral window needs to be employed. 
Table 6. DOAS equations considered in this study and corresponding AMFs. All DOAS equations have $\ln I_{\lambda}(k)$ in their left hand sides.

\begin{tabular}{lcc}
\hline DOAS equation & Right hand side & Air mass factor \\
\hline generalized & $-\int_{0}^{H} \mathcal{A}(\lambda, z) \sigma_{\lambda}(z) n(z) d z$ & Table 2 \\
DOAS & $+\ln \tilde{I}_{\lambda}(0)+P(\lambda)$ & \\
WFDOAS & $-V A(\lambda) \hat{\sigma}_{\lambda}+\ln \tilde{I}_{\lambda}(0)+P(\lambda)$ & Table 3 and 5 \\
MDOAS & $-V A(\lambda) \hat{\sigma}_{\lambda}+P(\lambda)$ & {$\left[\ln I_{\lambda}(0)-\ln I_{\lambda}(\bar{V})\right] / \hat{\sigma}_{\lambda} \bar{V}$} \\
standard DOAS & $-S \hat{\sigma}_{\lambda}+P(\lambda)$ & $A(\lambda) \longrightarrow A$, e.g., $A(\lambda) \hat{\sigma}_{\lambda}=A \hat{\sigma}_{\lambda}+P(\lambda)$ \\
\hline
\end{tabular}

\section{Conclusions}

Our comprehensive study shows that each DOAS equation represents a specific linear relationship between the intensity (normalized intensity) logarithm and a gaseous absorber amount. The specific characteristic of the DOAS equation is that contributions of scattering and reflection processes (Rayleigh scattering, aerosol extinction, surface albedo, etc.) into the intensity logarithm are approximated by a wavelength polynomial of a low order. This requires also gaseous absorption cross sections to contain a component rapidly varying with the wavelength which is not removed when subtracting the polynomial.

To derive a DOAS equation a linear relationship between the intensity logarithm and the gaseous absorber amount needs to be established. Whereas for observations of the direct solar light transmitted through the atmosphere a linear relationship follows immediately from the Beer-Lambert law, a linearization technique needs to be employed to obtain this relationship for observations of the scattered solar light with a non-negligible contribution of the multiple scattering. In this study we use the most general linearization approach based on the expansion of the intensity logarithm in the functional Taylor series with respect to a variation of the gaseous absorption coefficient. This allows us to derive the most general representation for the DOAS equation and introduce the appropriate air mass factor corresponding to an infinitesimal vertical path. In particular, we obtain that the air mass factor is expressed via the variational derivative of the intensity logarithm with respect to the gaseous absorption coefficient.

Using some approximations and simplifications we derive several DOAS equations which are summarized in Table 6. To simplify the representation we list DOAS equations for the vertical or slant column rather than for partial vertical columns. Furthermore, we do not indicate the dependence of all relevant variables on the observation/illumination conditions. To each DOAS equation an appropriate expression for the air mass factor is associated. For the standard DOAS equation, the expression provided for the AMF represents an example realization of the rule to map a wavelength depen- dent air mass factor to a constant value. There are also various alternative mapping rules suggested in previous publications.

Whereas all expressions for the air mass factor obtained for each particular DOAS equation are equivalent (see, e.g., Tables 3 and 5 for AMFs associated to the WFDOAS equation), the air mass factors obtained for different DOAS equations are not interchangeable. For example, a use of an air mass factor derived for the WFDOAS equation, see Table 3 , in combination with the MDOAS equation may lead to a significant retrieval error. Thus, a comparison of AMFs without any reference to the DOAS equation is meaningless. Instead, the pairs of DOAS equation and associated AMF have always to be considered.

In this study we demonstrate that in a case of a weak absorption all considered DOAS equations as well as corresponding expressions for the air mass factor become nearly equivalent. In particular, the air mass factor and the slant column can be considered to be nearly independent of the wavelength. A quantitative criterion of a weak gaseous absorption is suggested which is determined by a remaining non-linearity of the relationship between the intensity logarithm and gaseous absorber amount, see Eq. (108). This criterion can be used, for example, to check the validity of the standard DOAS equation for a given spectral window and observation/illumination conditions.

In a case of a non-weak absorption neither the DOAS equations nor the air mass factors are equivalent and one have always to ensure using appropriate combinations of the DOAS equation and the air mass factor. If treated properly, the correct solution for each particular observation setup can be obtained using any DOAS equation coupled with an appropriate AMF. However, sometimes an iterative process might be needed. Thus, selection of a particular DOAS equation for any practical application is rather a matter of the experience and available tools and is not directly related to the measurement setup. 


\section{Appendix A}

\section{Relationship between two forms of the standard DOAS equation}

In this section we demonstrate that the DOAS fit according to the standard DOAS equation in form of Eq. (39) results in the same slant column as using the DOAS equation written for the differential optical thickness and differential absorption cross section given by Eq. (46). To simplify further consideration let us introduce the vector-matrix notations as follows:

$\boldsymbol{y}=\left|\ln I_{\lambda_{1}}(k), \ldots, \ln I_{\lambda_{L}}(k)\right|^{\mathrm{T}}$,

$\boldsymbol{c}=\left|\sigma_{\lambda_{1}}, \ldots, \sigma_{\lambda_{L}}\right|^{\mathrm{T}}$,

i.e., vectors $y$ and $\boldsymbol{c}$ contain the values of the intensity $\log$ arithm and of the absorption cross section, respectively, at a discrete wavelength grid. The superscript $\mathrm{T}$ denotes the transposition. Using these notations Eq. (39) can be rewritten in the vector-matrix form as

$\boldsymbol{y}=-S \boldsymbol{c}+\mathbf{P} \boldsymbol{a}$,

where vector $\boldsymbol{a}$ contains the polynomial coefficients and matrix $\mathbf{P}$ is given by

$\mathbf{P}=\left[\begin{array}{ccccc}1 & \lambda_{1} & \lambda_{1}^{2} & \ldots & \lambda_{1}^{N} \\ \vdots & \vdots & \vdots & \vdots & \vdots \\ 1 & \lambda_{L} & \lambda_{L}^{2} & \ldots & \lambda_{L}^{N}\end{array}\right]$

Now let us write the differential absorption cross section introduced by Eq. (44) in the vector-matrix form as well:

$c^{d}=\boldsymbol{c}-\mathbf{P} \boldsymbol{a}_{\boldsymbol{c}}$.

Here, matrix $\mathbf{P}$ is given by Eq. (A4) and vector $\boldsymbol{a}_{\boldsymbol{c}}$ contains the polynomial coefficients. The latter are obtained minimizing the following quadratic form:

$\left\|\boldsymbol{c}-\mathbf{P} \boldsymbol{a}_{\boldsymbol{c}}\right\|^{2} \longrightarrow \min$.

The solution of this minimization problem is found as

$\boldsymbol{a}_{c}=\left[\mathbf{P}^{\mathrm{T}} \mathbf{P}\right]^{-1} \mathbf{P}^{\mathrm{T}} \boldsymbol{c}$.

Substituting the polynomial coefficients $\boldsymbol{a}_{c}$ given by Eq. (A7) into Eq. (A5), we have

$\boldsymbol{c}^{d}=\left\{\mathbf{I}-\mathbf{P}\left[\mathbf{P}^{\mathrm{T}} \mathbf{P}\right]^{-1} \mathbf{P}^{\mathrm{T}}\right\} \boldsymbol{c}$,

where $\mathbf{I}$ is the unity matrix. In exactly the same manner the following expression for the differential optical thickness is obtained:

$\boldsymbol{y}^{d}=\left\{\mathbf{I}-\mathbf{P}\left[\mathbf{P}^{\mathrm{T}} \mathbf{P}\right]^{-\mathbf{1}} \mathbf{P}^{\mathrm{T}}\right\} \boldsymbol{y}$.
Multiplying both sides of Eq. (A3) by the matrix I $\mathbf{P}\left[\mathbf{P}^{\mathrm{T}} \mathbf{P}\right]^{-1} \mathbf{P}^{\mathrm{T}}$ and taking into account Eqs. (A8) and (A9), we obtain

$\boldsymbol{y}^{d}=-S \boldsymbol{c}^{d}+\left\{\mathbf{I}-\mathbf{P}\left[\mathbf{P}^{\mathrm{T}} \mathbf{P}\right]^{-\mathbf{1}} \mathbf{P}^{\mathrm{T}}\right\} \mathbf{P} \boldsymbol{a}$.

As the second term in the right hand side of this equation is equal to zero, we have

$y^{d}=-S c^{d}$,

which is equivalent to Eq. (46) written in the vector-matrix form. As Eq. (A11) is obtained from Eq. (39), we can conclude that Eqs. (39) and (46) are equivalent as well.

\section{Appendix B}

\section{Derivation of alternative general expressions for the air mass factor}

In this section we derive alternative general expressions for the air mass factor containing the variational derivative of the intensity logarithm with respect to the absorber optical depth and vertical column as given by the third and fourth entries in Table 2, respectively. To this end we employ the functional Taylor series expansion of the intensity logarithm with respect to the variation of the absorber optical depth in exactly the same way as it was done before with respect to the absorption coefficient, see Eq. (50):

$\ln I_{\lambda}(k)=\ln I_{\lambda}(\bar{k})+\int_{0}^{H} \frac{\delta \ln I_{\lambda}}{\delta \tau_{\lambda}(z)} \delta \tau_{\lambda}(z) d z$.

Here, we omit the linearization error and define the absorption optical depth as

$\tau_{\lambda}(z)=\int_{z}^{H} k_{\lambda}\left(z^{\prime}\right) d z^{\prime}$.

We note that in contrast to the optical thickness which is defined as an integral over the entire atmosphere, see Eq. (3), the optical depth is not only a functional of the absorption coefficient but also a function of the altitude $z$. Clearly, a variation of the optical depth, $\delta \tau_{\lambda}(z)$, at an altitude level $z$ is given by

$\delta \tau_{\lambda}(z)=\int_{z}^{H} \delta k\left(z^{\prime}\right) d z^{\prime}$.

Substituting this expression into Eq. (B1) we have

$\ln I_{\lambda}(k)=\ln I_{\lambda}(\bar{k})+\int_{0}^{H} \frac{\delta \ln I_{\lambda}}{\delta \tau_{\lambda}(z)}\left[\int_{z}^{H} \delta k\left(z^{\prime}\right) d z^{\prime}\right] d z$. 
Introducing in the integral over $z^{\prime}$ the Heaviside step-function $H\left(z^{\prime}-z\right)$ we rewrite Eq. (B4) in the following form:

$\ln I_{\lambda}(k)=\ln I_{\lambda}(\bar{k})+\int_{0}^{H} \int_{0}^{H} \frac{\delta \ln I_{\lambda}}{\delta \tau_{\lambda}(z)} H\left(z^{\prime}-z\right) \delta k\left(z^{\prime}\right) d z^{\prime} d z$.

Exchanging the variables $z \leftrightarrow z^{\prime}$,

$\ln I_{\lambda}(k)=\ln I_{\lambda}(\bar{k})+\int_{0}^{H}\left[\int_{0}^{H} \frac{\delta \ln I_{\lambda}}{\delta \tau_{\lambda}\left(z^{\prime}\right)} H\left(z-z^{\prime}\right) d z^{\prime}\right] \delta k(z) d z$,

and excluding the Heaviside step-function we have

$\ln I_{\lambda}(k)=\ln I_{\lambda}(\bar{k})+\int_{0}^{H}\left[\int_{0}^{z} \frac{\delta \ln I_{\lambda}}{\delta \tau_{\lambda}\left(z^{\prime}\right)} d z^{\prime}\right] \delta k(z) d z$.

Comparing this equation to Eq. (58) and taking into account Eq. (74) we see that the representation for the air mass factor given by the third entry in Table 2 is derived.

Introducing the absorber vertical column between an altitude $z$ and the top of the atmosphere as

$V(z)=\int_{z}^{H} n\left(z^{\prime}\right) d z^{\prime}$

expanding then the intensity logarithm in the functional Taylor series with respect to the variation of $V(z)$ similar to Eq. (B1),

$\ln I_{\lambda}(k)=\ln I_{\lambda}(\bar{k})+\int_{0}^{H} \frac{\delta \ln I_{\lambda}}{\delta V(z)} \delta V(z) d z$,

and proceeding in exactly the same manner as was done to derive Eq. (B7), the following equation is obtained:

$\ln I_{\lambda}(k)=\ln I_{\lambda}(\bar{k})+\int_{0}^{H}\left[\int_{0}^{z} \frac{\delta \ln I_{\lambda}}{\delta V\left(z^{\prime}\right)} d z^{\prime}\right] \delta n(z) d z$.

Assuming that the variation of the absorption coefficient is caused only by a variation of the absorber number density, i.e., $\delta k(z)=\sigma_{\lambda} \delta n(z)$, the representation for the air mass factor given by the fourth entry in Table 2 is derived.

\section{Appendix C}

\section{Alternative expressions for the air mass factor associated to the WFDOAS equation}

In this section we derive alternative representations for the AMF associated to the WFDOAS equation which are equivalent to Eq. (85) and compare these to other expressions proposed in the literature. Using the partial scaling approximation for the vertical profile of the absorber number density as introduced in Sect. 6, the intensity logarithm can be considered as a function of partial vertical columns $V_{i}(i=$ $\left.1,2, \ldots, N_{L}\right)$, i.e., as a function of several scalar variables, rather than as a functional of the absorber number density profile. In this case a linear relationship between the intensity logarithm and partial vertical columns of the absorbing species is obtained employing the Taylor series expansion similar to Eq. (47):

$\ln I_{\lambda, j}(k)=\ln I_{\lambda, j}(\bar{k})+\sum_{i=1}^{N_{L}} \frac{\partial \ln I_{\lambda, j}}{\partial V_{i}} \Delta V_{i}$,

Here, $\partial \ln I_{\lambda, j} / \partial V_{i}$ is the partial derivative of the intensity logarithm with respect to the partial vertical column calculated at a priori column $\bar{V}_{i}$ and $\Delta V_{i}=V_{i}-\bar{V}_{i}$ is the variation of the partial vertical column in the $i$-th altitude layer. The linearization error is neglected. Using the Taylor series expansion given by Eq. (C1), an alternative representation for the layer-integrated weighting function introduced by Eq. (79) and consequently for the air mass factor can be obtained. To this end we rewrite the functional Taylor series for the intensity logarithm given by Eq. (58) as follows:

$\ln I_{\lambda, j}(k)=\ln I_{\lambda, j}(\bar{k})+\sum_{i=1}^{N_{L}} \int_{z_{i-1}}^{z_{i}} w_{\bar{k}, j}(\lambda, z) \delta k_{\lambda}(z) d z$,

where we neglect the linearization error, restore the subscript " $j$ " denoting different illumination/observation conditions, and represent the integral over the entire atmosphere as a sum of integrals over the discrete layers. Assuming the variation of the absorption coefficient to be caused only by a variation of the absorber number density, i.e., $\delta k_{\lambda}(z)=\sigma_{\lambda}(z) \delta n(z)$, and taking into account that according to Eq. (77) $\delta n(z)=$ $\Delta V_{i} \bar{n}(z) / \bar{V}_{i}$, we rewrite Eq. (C2) in the following equivalent form:

$$
\begin{aligned}
\ln I_{\lambda, j}(k)= & \ln I_{\lambda, j}(\bar{k}) \\
& +\sum_{i=1}^{N_{L}}\left[\frac{1}{\bar{V}_{i}} \int_{z_{i-1}}^{z_{i}} w_{\bar{k}, j}(\lambda, z) \sigma_{\lambda}(z) \bar{n}(z) d z\right] \Delta V_{i} .
\end{aligned}
$$

Comparing this equation to Eq. $(\mathrm{C} 1)$ the following relation is obtained:

$$
W_{i, j}(\lambda)=\frac{1}{\bar{V}_{i}} \int_{z_{i-1}}^{z_{i}} w_{\bar{k}, j}(\lambda, z) \sigma_{\lambda}(z) \bar{n}(z) d z=\frac{\partial \ln I_{\lambda, j}}{\partial V_{i}} .
$$

Thus, employing the partial scaling approximation, the layerintegrated weighting function is expressed via the partial derivative of the intensity logarithm with respect to the partial vertical column. For numerical calculations of the layerintegrated weighting function, the finite-difference approximation similar to Eq. (56) is appropriate:

$$
W_{i, j}(\lambda)=\frac{\ln I_{\lambda, j}\left(\bar{V}_{i}+\Delta V_{i}\right)-\ln I_{\lambda, j}\left(\bar{V}_{i}\right)}{\Delta V_{i}},
$$


where $\Delta V_{i}$ is a small variation of the absorber partial vertical column in the $i$-th atmospheric layer around its a priori value, $\bar{V}_{i}$. Taking into account the relationship between the box-AMF and the layer-integrated weighting function given by Eq. (83), the following expression for the box-AMF is obtained from Eq. (C4):

$A_{i, j}(\lambda)=-\left.\frac{1}{\hat{\sigma}_{\lambda}} \frac{\partial \ln I_{\lambda, j}}{\partial V_{i}}\right|_{\bar{V}_{i}}$,

where we notice explicity that the partial derivative is calculated at a priori partial vertical column, $\bar{V}_{i}$, and $\hat{\sigma}_{\lambda}$ is the vertical path weighted cross section as given by Eq. (24).

Introducing the layer optical thickness of the absorbing species as

$\tau_{i}(\lambda)=\int_{z_{i-1}}^{z_{i}} \sigma_{\lambda}(z) n(z) d z$,

and employing the partial scaling approximation as given by Eq. (77), we have

$\tau_{i}(\lambda)=\frac{V_{i}}{\bar{V}_{i}} \int_{z_{i-1}}^{z_{i}} \sigma_{\lambda}(z) \bar{n}(z) d z=\frac{V_{i}}{\bar{V}_{i}} \bar{\tau}_{i}(\lambda)$.

Thus,

$d \tau_{i}(\lambda)=d V_{i} \frac{\bar{\tau}_{i}(\lambda)}{\bar{V}_{i}}$

and Eq. (C6) can be rewritten in the following equivalent form:

$A_{i, j}(\lambda)=-\left.\frac{1}{\hat{\sigma}_{\lambda}} \frac{\partial \ln I_{\lambda, j}}{\partial \tau_{i}(\lambda)}\right|_{\bar{\tau}_{i}(\lambda)} \frac{\bar{\tau}_{i}(\lambda)}{\bar{V}_{i}}$.

In a case of a constant absorption cross section $\hat{\sigma}_{\lambda}=\sigma_{\lambda}$ and Eq. (C10) is simplified as

$A_{i, j}(\lambda)=-\left.\frac{\partial \ln I_{\lambda, j}}{\partial \tau_{i}(\lambda)}\right|_{\bar{\tau}_{i}(\lambda)}$,

where we take into account that $\bar{\tau}_{i}(\lambda)=\sigma_{\lambda} \bar{V}_{i}$.

A similar expression has been suggested by Slusser et al. (1996) for the box-AMF and later by Stammes and Koelemeijer (1999) for the total AMF. In these publications the numerical calculations have been performed employing the finite-difference representation of Eq. (C11), e.g., the following formula has been used by Slusser et al. (1996):

$A_{i, j}(\lambda)=-\frac{\Delta\left(\ln I_{\lambda, j}\right)}{\Delta \tau_{i}(\lambda)}=-\frac{\Delta\left(\ln I_{\lambda, j}\right)}{\sigma_{\lambda} \Delta V_{i}}$,

where $\Delta V_{i}$ is an enhancement of the partial vertical column in the $i$-th atmospheric layer.

Having derived an equation for the box-AMF we can easily obtain the corresponding expression for the total AMF using Eq. (31). For example, considering the box-AMF given by Eq. (C10) the following equation for the total AMF is obtained:

$A_{j}(\lambda)=-\frac{1}{\bar{\tau}(\lambda)} \sum_{i=1}^{N_{L}}\left[\left.\frac{\partial \ln I_{\lambda, j}}{\partial \tau_{i}(\lambda)}\right|_{\bar{\tau}_{i}(\lambda)} \bar{\tau}_{i}(\lambda)\right]$,

where we take into account that, according to Eq. (24), $\bar{\tau}(\lambda)=\hat{\sigma}_{\lambda} \bar{V}$. Assuming each altitude layer to be vertically homogeneous, the layer optical thickness of the absorbing species can be expressed as $\bar{\tau}_{i}(\lambda)=\sigma_{\lambda, i} \bar{n}_{i} \Delta z_{i}$, where the absorber cross section, $\sigma_{\lambda, i}$, and the absorber number density, $\bar{n}_{i}$, are constant within the $i$-th altitude layer having a geometrical thickness $\Delta z_{i}$. Thus, Eq. (C13) results in

$A_{j}(\lambda)=-\frac{1}{\bar{\tau}(\lambda)} \sum_{i=1}^{N_{L}}\left[\left.\frac{\partial \ln I_{\lambda, j}}{\partial \tau_{i}(\lambda)}\right|_{\bar{\tau}_{i}(\lambda)} \sigma_{\lambda, i} \bar{n}_{i} \Delta z_{i}\right]$.

This equation coincides with the expression the for the total air mass factor in a discrete representation proposed by Palmer et al. (2001).

Acknowledgements. We thank Andreas Richter and Folkard Wittrock for a fruitful discussion. This work has been funded in parts by the German Aerospace Center DLR (grant 50EE0727), the University and the Land Bremen.

Edited by: B. Funke

\section{References}

Bovensmann, H., Burrows, J. P., Buchwitz, M., Frerick, J., Noël, S., Rozanov, V. V., Chance, K. V., and Goede, A. P. H.: SCIAMACHY: Mission objectives and measurement modes, J. Atmos. Sci., 56, 127-149, 1999.

Brewer, A. W., McElroy, C. T., and Kerr, J. B.: Nitrogen dioxide concentrations in the atmosphere, Nature, 246, 129-133, 1973.

Bruns, M., Buehler, S. A., Burrows, J. P., Heue, K.-P., Platt, U., Pundt, I., Richter, A., Rozanov, A., Wagner, T., and Wang, P.: Retrieval of Profile Information from Airborne Multi Axis UV/visible Skylight Absorption Measurements, Appl. Optics, 43, 4415-4426, 2004.

Bruns, M., Buehler, S. A., Burrows, J. P., Richter, A., Rozanov, A., Wang, P., Heue, K. P., Platt, U., Pundt, I., and Wagner, T.: $\mathrm{NO}_{2}$ Profile retrieval using airborne multi axis UV-visible skylight absorption measurements over central Europe, Atmos. Chem. Phys., 6, 3049-3058, doi:10.5194/acp-6-3049-2006, 2006.

Buchwitz, M., Rozanov, V. V., and Burrows, J. P.: A near-infrared optimized DOAS method for the fast global retrieval of atmospheric $\mathrm{CH}_{4}, \mathrm{CO}, \mathrm{CO}_{2}, \mathrm{H}_{2} \mathrm{O}$, and $\mathrm{N}_{2} \mathrm{O}$ total column amounts from SCIAMACHY Envisat-1 nadir radiances, J. Geophys. Res., D105, 15231-15245, 2000.

Burrows, J. P., Weber, M., Buchwitz, M., Rozanov, V., enmayer, A. L.-W., Richter, A., DeBeek, R., Hoogen, R., Bramstedt, K., Eichmann, K.-U., Eisinger, M., and Perner, D.: The Global Ozone Monitoring Experiment (GOME): Mission concept and first scientific results, J. Atmos. Sci., 56, 151-175, 1999.

Coldewey-Egbers, M., Weber, M., Lamsal, L. N., de Beek, R., Buchwitz, M., and Burrows, J. P.: Total ozone retrieval from 
GOME UV spectral data using the weighting function DOAS approach, Atmos. Chem. Phys., 5, 1015-1025, doi:10.5194/acp5-1015-2005, 2005.

Deepak, A.: Inversion methods in atmospheric remote sounding, Academic Press, 1977.

Diebel, D., Burrows, J., Beek, R. D., Munro, R., Kerridge, B., Frank, H., Platt, U., and Marquard, L.: Tracegas study. Detailed analysis of the retrieval algorithms selected for the level 1-2 processing of GOME data, Progress report 2, ESA Contract 10728/94/NL/CN, 1994.

Fish, D., Jones, R., and Strong, E.: Midlatitude observations of the diurnal variation of stratospheric BrO, J. Geophys. Res., 100, 18863-18871, 1995.

Frankenberg, C., Platt, U., and Wagner, T.: Iterative maximum a posteriori (IMAP)-DOAS for retrieval of strongly absorbing trace gases: Model studies for $\mathrm{CH}_{4}$ and $\mathrm{CO}_{2}$ retrieval from near infrared spectra of SCIAMACHY onboard ENVISAT, Atmos. Chem. Phys., 5, 9-22, doi:10.5194/acp-5-9-2005, 2005.

Haley, C. S., Brohede, S. M., Sioris, C. E., Griffioen, E., Murtagh, D. P., McDade, I. C., Eriksson, P.,Llewellyn, E. J., Bazureau, A., and Goutail, F.: Retrieval of stratospheric O3 and NO2 profiles from Odin Optical Spectrograph and Infrared Imager System (OSIRIS) limb-scattered sunlight measurements, J. Geophys. Res, 109(D16), 303, doi:10.1029/2004JD004588, 2004.

Harrison, A.: Midsummer stratospheric $\mathrm{NO}_{2}$ at latitude $45^{\circ} \mathrm{S}$, Can. J. Phys., 57, 1110, doi:10.1139/p79-155, 1979.

Hazewinkel, M. (Ed.): Encyclopaedia of Mathematics, SpringerVerlag Berlin Heidelberg New York, 2002.

Hendrick, F., Barret, B., Van Roozendael, M., Boesch, H., Butz, A., De Mazière, M., Goutail, F., Hermans, C., Lambert, J.C., Pfeilsticker, K., and Pommereau, J.-P.: Retrieval of nitrogen dioxide stratospheric profiles from ground-based zenith-sky UV-visible observations: validation of the technique through correlative comparisons, Atmos. Chem. Phys., 4, 2091-2106, doi:10.5194/acp-4-2091-2004, 2004.

Hönninger, G., von Friedeburg, C., and Platt, U.: Multi axis differential optical absorption spectroscopy (MAX-DOAS), Atmos. Chem. Phys., 4, 231-254, doi:10.5194/acp-4-231-2004, 2004.

Hoogen, R., Rozanov, V. V., and Burrows, J. P.: Ozone profiles from GOME satellite data: Algorithm description and first validation, J. Geophys. Res., 104, 8263-8280, doi:10.1029/1998JD100093, 1999.

Klenk, K. F., Bhartia, P. K., Kaveeshwar, V. G., McPeters, R. D., Smith, P. M., and Fleig, A. J.: Total Ozone Determination from the Backscattered Ultraviolet (BUV), J. Appl. Meteorol., 21, 1672-1684, 1982.

Marquard, L., Wagner, T., and Platt, U.: Improved air mass factor concepts for scattered radiation differential optical absorption spectroscopy of atmospheric species, J. Geophys. Res., 105, 1315-1327, 2000.

McKenzie, R., Johnston, P., McElroy, C., Kerr, J., and Solomon, S.: Altitude distributions of stratospheric components from groundbased measurements at twilight, J. Geophys. Res., 96, 15499$15511,1991$.

McKenzie, R. L. and Johnston, P. V.: Seasonal variations in stratospheric $\mathrm{NO}_{2}$ at $45^{\circ} \mathrm{S}$, Geophys. Res. Lett., 9, 1255-1258, 1982.

Noxon, J., Whipple, E., and Hyde, R.: Stratospheric $\mathrm{NO}_{2}$ 1. Observational Method and Behavior at Mid-Latitude, J. Geophys. Res., 84, 5047-5065, 1979.
Palmer, P., Jacob, D., Chance, K., Martin, R., Spurr, R. J. D., Kurosu, T., Bey, I., Ytantosca, R., Fiore, A., and Li, Q.: Air mass factor formulation for spectroscopic measurements from satellites: Application to formaldehyde retrievals from the Global Ozone Monitoring Experiment, J. Geophys. Res., 106, 1453914550, 2001.

Perliski, L. and Solomon, S.: On the evaluation of air mass factors for atmospheric near-ultraviolet and visible absorption spectroscopy, J. Geophys. Res., 98, 10363-10374, 1993.

Platt, U.: Air Monitoring by Spectroscopic Techniques, chap. Differential optical absorption spectroscopy (DOAS), John Wiley, New York, 1994.

Platt, U. and Perner, D.: Direct measurements of atmospheric $\mathrm{CH}_{2} \mathrm{O}, \mathrm{HNO}_{2}, \mathrm{O}_{3}, \mathrm{NO}_{2}$, and $\mathrm{SO}_{2}$ by differential optical absorption in the near UV, J. Geophys. Res., 85, 7453-7458, 1980.

Platt, U. and Stutz, J.: Differential optical absorption spectroscopy. Principles and Applications., Springer-Verlag Berlin Heidelberg, 2008.

Postylyakov, O., Belikov, I., Elansky, N., and Elokhov, A.: Observations of the ozone and nitrogen dioxide profiles in the TROICA-4 experiment, Adv. Space Res., 37, 2231-2237, 2006.

Puķīe, J., Kühl, S., Deutschmann, T., Platt, U., and Wagner, T. Extending differential optical absorption spectroscopy for limb measurements in the UV, Atmos. Meas. Tech., 3, 631-653, doi:10.5194/amt-3-631-2010, 2010.

Richter, A.: Absorptionsspectroscopishce Messungen stratosphärischer Spurengase über Bremen, $53^{\circ} \mathrm{N}, \mathrm{PhD}$ thesis, Fachbereich Physik der Universität Bremen, 1997.

Richter, A., Eisinger, M., Enmayer, A. L.-W., and Burrows, J. P.: DOAS zenith sky observations: 2 . Seasonal variation of $\mathrm{BrO}$ over Bremen $\left(53^{\circ}\right.$ N) 1994-1995, J. Atmos. Chem., 32, 83-99, 1999.

Rodgers, C. D.: Inverse methods for atmospheric sounding, World Scientific, 2000.

Roscoe, H. K., Johnston, P. V., Van Roozendael, M., et al.: Slant column measurements of $\mathrm{O}_{3}$ and $\mathrm{NO}_{2}$ during the NDSC intercomparison of zenith-sky UV-visible spectrometers in June 1996, J. Atmos. Chem., 32, 281-314, 1999.

Rozanov, A.: SCIATRAN 2.X: Radiative transfer model and retrieval software package, online available at: http://www.iup. physik.uni-bremen.de/sciatran, 2008.

Rozanov, A., Bovensmann, H., Bracher, A., Hrechanyy, S., Rozanov, V., Sinnhuber, M., Stroh, F., and Burrows, J.: $\mathrm{NO}_{2}$ and $\mathrm{BrO}$ vertical profile retrieval from SCIAMACHY limb measurements: Sensitivity studies, Adv. Space Res., 36, 846-854, doi:10.1016/j.asr.2005.03.013, 2005a.

Rozanov, A., Rozanov, V., Buchwitz, M., Kokhanovsky, A., and Burrows, J. P.: SCIATRAN 2.0 - A new radiative transfer model for geophysical applications in the $175-2400 \mathrm{~nm}$ spectral region, Adv. Space Res., 36, 1015-1019, 2005b.

Rozanov, V. V.: Light Scattering Reviews, vol. 1, chap. Adjoint radiative transfer equation and inverse problems, Berlin: Springer, 339-392, 2006.

Rozanov, V. V. and Kokhanovsky, A. A.: Light Scattering Reviews, vol. 3, chap. Impact of single- and multi-layered cloudiness on ozone vertical column retrievals using nadir observations of backscattered solar radiation, 133-189, Berlin: Springer, 2008.

Rozanov, V. V. and Rozanov, A. V.: Relationship between different approaches to derive weighting functions related to atmospheric 
remote sensing problems, J. Quant. Spectrosc. Ra., 105, 217242, 2007.

Rozanov, V. V., Kurosu, T., and Burrows, J.: Retrieval of atmospheric constituents in the UV-Visible: A new quasi-analytical approach for the calculation of weighting functions, J. Quant. Spectrosc. Ra., 60, 277-299, 1998.

Rozanov, V. V., Rozanov, A. V., and Kokhanovsky, A. A.: Derivatives of the radiation field and their application to the solution of inverse problems, in: Light Scattering Reviews, edited by Kokhanovsky, A. A., vol. 2, Berlin: Springer, 205-265, 2007.

Sarkissian, A., Roscoe, H. K., and Fish, D. J.: Ozone measurements by zenith-sky spectrometers: an evaluation of errors in air-mass factors calculated by radiative transfer models, J. Quant. Spectrosc. Ra., 54, 471-480, 1995.

Slusser, J., Hammond, K., Kylling, A., Stamnes, K., Perliski, L., Dahlback, A., Anderson, D., and DeMajistre, R.: Comparison of air mass computations, J. Geophys. Res., 101, 9315-9321, 1996.

Solomon, S., Schmeltekopf, A. L., and Sanders, R. W.: On the interpretation of zenith sky absorption measurements, J. Geophys. Res., 92, 9315-9321, 1987.

Stammes, P. and Koelemeijer, R. B. A.: Error analysis of the GOME ozone column retrieval method, in: Proc. European Symposium on Atmospheric Measurements from Space, ESTEC, Noordwijk, The Netherlands, 201-207, 1999.

Thomas, G. E. and Stamnes, K.: Radiative transfer in the atmosphere and ocean, Cambridge University Press, 1999.
Volterra, V.: Theory of functionals and of integral and integrodifferential equations, Dover, New York, 1959.

Wagner, T., Burrows, J. P., Deutschmann, T., Dix, B., von Friedeburg, C., Frieß, U., Hendrick, F., Heue, K.-P., Irie, H., Iwabuchi, H., Kanaya, Y., Keller, J., McLinden, C. A., Oetjen, H., Palazzi, E., Petritoli, A., Platt, U., Postylyakov, O., Pukite, J., Richter, A., van Roozendael, M., Rozanov, A., Rozanov, V., Sinreich, R., Sanghavi, S., and Wittrock, F.: Comparison of box-airmass-factors and radiances for Multiple-Axis Differential Optical Absorption Spectroscopy (MAX-DOAS) geometries calculated from different UV/visible radiative transfer models, Atmos. Chem. Phys., 7, 1809-1833, doi:10.5194/acp-7-1809-2007, 2007.

Weber, M., Lamsal, L. N., Coldewey-Egbers, M., Bramstedt, K., and Burrows, J. P.: Pole-to-pole validation of GOME WFDOAS total ozone with groundbased data, Atmos. Chem. Phys., 5, 1341-1355, doi:10.5194/acp-5-1341-2005, 2005.

Widom, H.: Lectures on integral equations, Amer. Book Comp., 1969.

Zabreiko, P. P., Koshelev, A. I., Krasnoselskii, M. A., Mikhlin, S. G., Rakovshchik, L. S., Stet'senko, V. Y., Shaposhnikova, T. O., and Anderssen, R. S., eds.: Integral equations - a reference text, chap. 2, Noordhoff, translated from Russian, 1958. 